TRANSACTIONS OF THE

AMERICAN MATHEMATICAL SOCIETY

Volume 353, Number 8, Pages 3149-3180

S 0002-9947(01)02643-5

Article electronically published on January 18, 2001

\title{
ON THE COMMUTATION OF THE TEST IDEAL WITH LOCALIZATION AND COMPLETION
}

\author{
GENNADY LYUBEZNIK AND KAREN E. SMITH
}

\begin{abstract}
Let $R$ be a reduced ring that is essentially of finite type over an excellent regular local ring of prime characteristic. Then it is shown that the test ideal of $R$ commutes with localization and, if $R$ is local, with completion, under the additional hypothesis that the tight closure of zero in the injective hull $E$ of the residue field of every local ring of $R$ is equal to the finitistic tight closure of zero in $E$. It is conjectured that this latter condition holds for all local rings of prime characteristic; it is proved here for all Cohen-Macaulay singularities with at most isolated non-Gorenstein singularities, and in general for all isolated singularities. In order to prove the result on the commutation of the test ideal with localization and completion, a ring of Frobenius operators associated to each $R$-module is introduced and studied. This theory gives rise to an ideal of $R$ which defines the non-strongly F-regular locus, and which commutes with localization and completion. This ideal is conjectured to be the test ideal of $R$ in general, and shown to equal the test ideal under the hypothesis that $0_{E}^{*}=0_{E}^{f g *}$ in every local ring of $R$.
\end{abstract}

\section{INTRODUCTION}

Tight closure, first introduced by Hochster and Huneke over ten years ago, has become an active branch of commutative algebra. It has numerous applications both within commutative algebra and to closely related areas. For example, it is useful in studying Cohen-Macaulayness and questions of uniform behavior in Noetherian rings, as well as in studying singularities and vanishing theorems for algebraic varieties.

Tight closure is a closure operation performed on ideals in a Noetherian commutative ring containing a field; the definition involves reduction to prime characteristic and iteration of the Frobenius map. Despite intense study, many basic questions about tight closure remain unanswered. Most insidious among these is our failure to have ascertained that the theory is well behaved under localization. For example, given an ideal $I$ in a ring $R$, it is not known whether or not the image of its tight closure $I^{*} R_{P}$ in a local ring $R_{P}$ of $R$ is the same as the tight closure $\left(I R_{P}\right)^{*}$ of its image in $R_{P}$. Related issues, such as the preservation of the property that all ideals are tightly closed under localization, are likewise open.

Received by the editors January 4, 1999 and, in revised form, July 12, 1999 and March 25, 2000.

1991 Mathematics Subject Classification. Primary 13A35; Secondary 13C99.

Key words and phrases. Tight closure, test ideal, localisation, Frobenius action.

Both authors were supported by the National Science Foundation.

(C)2001 American Mathematical Society 
Test elements are elements that simultaneously annihilate all tight closure relations. They form an essential aspect of the theory of tight closure, but again, their behavior under localization and completion has remained largely mysterious. The failure to understand the behavior of test elements (and tight closure in general) under completion and localization has led to an unfortunate abundance of technical terms designed to accommodate this problem. For example, although we fully expect that a test element remains a test element after localization and completion, we must define a completely stable test element to be an element whose image in every local ring and in every completed local ring of $R$ is a test element.

The purpose of this paper is to develop a theory for treating the localization and the completion problems for test elements, and then use this theory to resolve these problems in some broad classes of rings.

We will identify a natural ideal $\tilde{\tau}$ of a Noetherian ring of prime characteristic, that is contained in the ideal generated by all completely stable test elements (the completely stable test ideal). For rings essentially of finite type over an excellent regular local ring of prime characteristic, we will show that $\tilde{\tau}$ is well behaved under localization and under completion, and that $\tilde{\tau}$ defines the non-strongly F-regular locus (see Definition 2.7). We conjecture that $\tilde{\tau}$ is the test ideal (and hence, is also the completely stable test ideal) and prove that this is so for Cohen-Macaulay rings with only isolated non-Gorenstein points, for rings with only isolated singularities, and for $\mathbb{N}$-graded rings. Using recent work of Aberbach and MacCrimmon [AM], we deduce that $\tilde{\tau}$ is the test ideal also for Cohen-Macaulay $\mathbb{Q}$-Gorenstein rings. It follows in all these cases that every test element is a completely stable test element.

Our method for defining $\tilde{\tau}$ is based on our theory of Frobenius structures on a module $M$ over a ring of prime characteristic, which we think is quite interesting in its own right. We introduce a (non-commutative) subring $\mathcal{F}(M)$ of the ring of all additive self-maps $\operatorname{End}_{\mathbb{Z}}(M)$ of $M$, the ring of Frobenius operators on $M$. We study modules over this ring, with particular emphasis on the structure of $M$ as an $\mathcal{F}(M)$-module. The case where $M$ is the injective hull $E$ of the residue field of a local ring plays a central role. For example, when $R$ is a complete local domain, $E$ turns out to have a unique maximal proper $\mathcal{F}(E)$-submodule and our ideal $\tilde{\tau}$ is defined as its annihilator.

This idea generalizes the attack on the localization problem for the test ideal of a Gorenstein ring launched in [S3]. In a Gorenstein ring, the injective hull of the residue field is isomorphic to a local cohomology module, and therefore comes equipped with a natural action of Frobenius. This puts a natural $R\{F\}$-module structure on $E$, where $R\{F\}$ is the subring of $\operatorname{End}_{\mathbb{Z}}(R)$ generated by $R$ (acting on itself by multiplication) and by the Frobenius operator $F$. For Gorenstein local rings whose completion is a domain, it was shown that the $R\{F\}$-module $E$ has a unique maximal proper submodule and that its annihilator in $R$ is the test ideal. Using this $R\{F\}$-module structure, the test ideal of a complete local Gorenstein domain was shown to behave well under localization. The methods of [S3] break down for non-Gorenstein rings because, in general, there does not seem to be a natural $R\{F\}$ structure on $E$. The solution we present in this paper is to consider all $R\{F\}$-module structures on $E$. These form a ring $\mathcal{F}(E)$ in a natural way which acts on $E$, and when $R$ is Gorenstein, $\mathcal{F}(E)=R\{F\}$. Again, if $\hat{R}$ is a domain, there is a unique maximal proper $\mathcal{F}(E)$-submodule of $E$ whose annihilator behaves well under localization and completion. We prove that this annihilator is the test ideal in the cases described above, and conjecture this to be true always. 
We will describe our results in more detail in the next section, while simultaneously introducing the notation, definitions, and preliminary results we will use throughout the paper.

The authors would like to thank Florian Enescu, who made us aware of Radu's paper $[\mathrm{R}$, and Yongwei Yao, who saved us from a gap in the arguments by observing the omission of the $S_{2}$ hypothesis in Example 3.7, which affected the statements of later results.

\section{Definitions, notation and PRECise Statements OF TIGHT CLOSURE RESULTS}

Throughout this paper $R$ is a commutative Noetherian ring containing a field of prime characteristic $p>0$.

The Frobenius map $R \stackrel{F}{\longrightarrow} R$ is the map which sends an element $r$ to its $p^{\text {th }}$-power $r^{p}$. If the Frobenius map is finite, we say that $R$ is $F$-finite. This weak condition is preserved by localization and by finitely generated algebra extension. Examples of F-finite rings include any algebra essentially of finite type over a perfect (or even Ffinite) field, or any complete local ring with an F-finite residue field. Every F-finite ring is excellent $\mathrm{Ku}$.

We denote by $R^{(e)}$ the $R$-bimodule whose underlying abelian group is that of $R$, whose left $R$-module structure is the usual one and whose right $R$-module structure is defined via Frobenius: $r$ in $R$ acts on $x$ in $R^{(e)}$ by $r \cdot x=r x$ on the left and by $x \cdot r=r^{p^{e}} x$ on the right. If $M$ is an $R$-module, we consider $R^{(e)} \otimes_{R} M$ as a left $R$-module via the left $R$-module structure on $R^{(e)}$.

We quickly recall some of the basic definitions we need from the theory of tight closure. The reader is referred to Hochster and Huneke's fundamental paper [HH1] for more information.

2.1. Definition. Let $N \stackrel{\imath}{\hookrightarrow} M$ be an inclusion of a submodule $N$ in an $R$-module $M$.

(1) The tight closure, $N_{M}^{*}$, of $N$ in $M$ is the submodule of $M$ consisting of all elements $x \in M$ for which there exists $c \in R$ not contained in any minimal prime of $R$ such that $c \otimes_{R} x \in \operatorname{Im}\left(R^{(e)} \otimes_{R} N \stackrel{\operatorname{id} \otimes_{\imath}}{\longrightarrow} R^{(e)} \otimes_{R} M\right)$ for all $e \gg 0$.

(2) The finitistic tight closure, $N_{M}^{* f g}$, of $N$ in $M$ is the union of the tight closures of $N \cap M^{\prime}$ in $M^{\prime}$, as $M^{\prime}$ ranges over all the finitely generated submodules $M^{\prime}$ of $M$.

In the definition of tight closure, the element ' $c$ ' that is used to test tight closure can depend on $x, M$ and $N$. An important feature of the theory of tight closure is that, in fact, there exist "test elements" that can be used in any tight closure test for finitely generated modules.

2.2. Definition. (1) The test ideal of $R$, denoted $\tau(R)$ or just $\tau$ when $R$ is clear from context, consists of all elements $c \in R$ that annihilate $N_{M}^{*} / N$ for all finitely generated $M$ and all submodules $N$ of $M$.

(2) The completely stable test ideal of $R$ consists of all elements $c \in R$ whose image in every local ring of $R$ and in the completion of every local ring of $R$ (at its unique maximal ideal) belongs to the test ideal of the respective ring.

(3) A test element (respectively, completely stable test element) is an element of the test ideal (respectively, completely stable test ideal) that is not in any minimal prime of $R$. 
Completely stable test elements exist for any reduced $F$-finite ring [HH2], and even for any reduced ring essentially of finite type over an excellent local ring [HH3]. One cannot expect to find test elements in a non-reduced ring. For example, if $(R, m)$ is a zero dimensional local ring that is not reduced, then it is easy to check that the tight closure of the zero ideal is $m$; in particular, the test ideal must be a proper ideal, but since every non-unit is contained in a minimal prime, there are no test elements.

We denote by $E_{R}$, or simply by $E$ when $R$ is understood, the direct sum of the injective hulls of the modules $R / m$ as $m$ ranges through all the maximal ideals of $R$. In particular, if $R$ is local, $E_{R}$ is the injective hull of the residue field of $R$. The finitistic tight closure of the zero module in $E$ plays a distinguished role in the theory of tight closure because its annihilator in $R, \operatorname{Ann}_{R}\left(0_{E}^{* f g}\right)$, is the test ideal of $R$ [HH1, 8.23].

In this paper we study the tight closure $0_{E}^{*}$ of the zero module in $E$. We expect that this module is the same as the finitistic tight closure module $0_{E}^{* f g}$. We denote its annihilator by $\tilde{\tau}$, or by $\tilde{\tau}(R)$ when we want to emphasize the ring. The ideal $\tilde{\tau}$ is clearly contained in the test ideal of $R$, since $0_{E}^{* f g} \subset 0_{E}^{*}$, and we expect that it is equal to the test ideal. That $\tau=\tilde{\tau}$ is known for Gorenstein rings [S2, 3.3], for localizations of $\mathbb{N}$-graded rings at the unique homogeneous maximal ideal [LS], and, as we show in Section 8, for Cohen-Macaulay rings Gorenstein on the punctured spectrum and for isolated singularities.

In Section 5, we prove that $\tilde{\tau}$ behaves well under localization and completion. For example, as a special case of Theorem 7.2 we have:

2.3. Theorem. Let $R$ be a reduced ring essentially of finite type over an excellent regular local ring of prime characteristic. For every multiplicative set $S$ of $R$, $\tilde{\tau}(R) R\left[S^{-1}\right]=\tilde{\tau}\left(R\left[S^{-1}\right]\right)$. Furthermore, for any prime ideal $P, \tilde{\tau}(R) \widehat{R_{P}}=\tilde{\tau}\left(\widehat{R_{P}}\right)$, where $\widehat{R_{P}}$ is the completion of the local ring $R_{P}$ at its unique maximal ideal.

This has an important consequence for the test ideal:

2.4. Corollary. Let $R$ be a reduced local ring that is a homomorphic image of an excellent regular local ring of prime characteristic, and assume that $0_{E}^{*}=0_{E}^{* f g}$. Then the test ideal commutes with completion. More precisely, the expansion of the test ideal of $R$ to $\hat{R}$ is the test ideal of the complete local ring $\hat{R}$. Furthermore, the test ideal is identical to the completely stable test ideal.

Hochster and Huneke had conjectured [HH1, p. 85] that local rings which admit test elements are " $\tau$-complete," meaning that $\tau(R) \hat{R}=\tau(\hat{R})$. What we show in Theorem 7.2 is that $\tilde{\tau}(R) \hat{R}=\tilde{\tau}(\hat{R})$, so the theorem above follows whenever $\tau$ and $\tilde{\tau}$ are equal. Thus we settle the conjecture of Hochster and Huneke, at least in the cases where we know $0_{E}^{*}=0_{E}^{* f g}$. In the final section of the paper, we identify classes of rings for which we have been able to show that $0_{E}^{*}=0_{E}^{* f g}$, including rings with isolated singularities and Cohen-Macaulay local rings with isolated nonGorenstein points. This is also known for localizations of $\mathbb{N}$-graded rings at the unique homogeneous maximal ideal [LS] and for Cohen-Macaulay rings that admit a canonical module defining a torsion element in the divisor class group [AM].

Some caution is in order in trying to conclude that the test ideal commutes with localization in a similar way. Even if we know that $\tilde{\tau}(R)=\tau(R)$, it does not follow 
immediately that $\tilde{\tau}\left(R_{P}\right)=\tau\left(R_{P}\right)$, even if we know that $\tilde{\tau}$ is well behaved under localization. However, we do have the following corollary:

2.5. Corollary. Let $R$ be a reduced ring essentially of finite type over an excellent regular local ring of prime characteristic. If, for each prime ideal $P$ of $R$, the tight closure of the zero module in the injective hull of the residue field of $R_{P}$ (computed over $R_{P}$ ) is equal to its finitistic tight closure, then the test ideal of $R$ commutes with localization.

These results point to the importance of the following conjecture.

2.6. Conjecture. If $N$ is a submodule of an Artinian module $M$ over an excellent local ring, then the tight closure of $N$ in $M$ is the union of the tight closures of $N \cap M^{\prime}$ in $M^{\prime}$, where $M^{\prime}$ ranges over all finitely generated submodules of $M$.

This paper shows that settling this conjecture, even in the case where $N$ is the zero module and $M$ is the injective hull of the residue field of a local ring $R$, would completely settle the issue regarding the behavior of the test ideal under localization and completion, at least for rings essentially of finite type over an excellent regular local ring of prime characteristic. In particular, it would show that every test element is a completely stable test element, and it would establish that weak and strong F-regularity are equivalent for F-finite rings, and hence also that weak Fregularity is preserved by localization. We recall the definitions of strong and weak F-regularity.

2.7. Definition. Let $R$ be a Noetherian ring of prime characteristic $p$.

(1) The ring $R$ is said to be weakly $F$-regular if all ideals are tightly closed.

(2) The ring $R$ is said to be strongly $F$-regular if it is reduced, F-finite, and for all elements $c$ not in any minimal prime of $R$, there exists a positive integer $e$ such that the $R$-module map $R \rightarrow R^{1 / p^{e}}$ sending 1 to $c^{1 / p^{e}}$ splits.

It is not hard to see that strongly F-regular rings are weakly F-regular, and it is conjectured that they are equivalent in the F-finite case. To show this equivalence would be an important breakthrough because it would finally establish that the property of weak F-regularity is preserved under localization. The concept of strong F-regularity was introduced in [ $\mathrm{HH} 2$, where all these facts are proved. The equivalence is known in a number of cases: for Gorenstein rings [HH2], for Cohen-Macaulay rings with only isolated non- $\mathbb{Q}$-Gorenstein points $[\mathrm{Mac}$, for rings of dimension three or less $[\mathrm{W}]$, and for $\mathbb{N}$-graded rings over a field [LS].

The property of strong F-regularity can be characterized easily in terms of the theory of Frobenius structures developed here. In Theorem 4.1, we show that a reduced F-finite local ring is strongly F-regular if and only if the injective hull $E$ of its residue field is a simple $\mathcal{F}(E)$-module.

2.8. Remark. The concept of strong F-regularity can be enlarged so as to avoid the assumption that $R$ is $\mathrm{F}$-finite. One could simply require that the map $R \rightarrow R^{1 / p^{e}}$ sending 1 to $c^{1 / p^{e}}$ is pure, rather than split. (In general, splitting of a map is equivalent to purity when the cokernel is finitely presented [Mats, p. 54].) However, with this definition, it is not clear that $R$ is strongly F-regular if and only if for each maximal ideal $m$ of $R, R_{m}$ is strongly F-regular. We get around this problem by defining a ring to be strongly F-regular if its localizations at each maximal ideal are strongly F-regular, and then by defining strong F-regularity for a local ring in 
terms of the purity of the appropriate map. As shown in [S1, 7.1.2], an equivalent characterization of this enlarged definition can be given in terms of tight closure:

2.9. Proposition ([S1, 7.1.2]). Let $(R, m)$ be a local ring of prime characteristic $p$. Then the following are equivalent:

(1) For all $c$ not in any minimal prime, there exists a positive integer e such that the map $R \rightarrow R^{(e)}$ sending 1 to $c$ is pure as a right $R$-module map.

(2) The zero module is tightly closed in the injective hull of the residue field of $R$.

Remark. If $R$ is reduced, the purity of the map $R \rightarrow R^{(e)}$ as a right $R$-module map is equivalent to the purity of the map $R \rightarrow R^{1 / p^{e}}$ sending 1 to $c^{1 / p^{e}}$. So if $R$ is reduced and F-finite, the two conditions above are equivalent to the strong F-regularity of $R$.

Because we make substantial use of this fact, we include a proof for the convenience of the reader, a slicker proof than the original in [S1, 7.1.2].

Proof. If $M \stackrel{\phi}{\longrightarrow} N$ is a map of finitely generated modules over a Noetherian local ring $R$, then the purity of $\phi$ is equivalent to the injectivity of the map

$$
M \otimes E \stackrel{\phi \otimes i d}{\longrightarrow} N \otimes E
$$

where $E$ is the injective hull of the residue field of $R$ [HH4, 2.1e]. Now, if a pure map exists as in (1) for some fixed $e_{0}$, then such a pure map exists also for all $e>e_{0}$ (for the same value of $c$ ). So if (1) holds, then the map $E \rightarrow R^{(e)} \otimes E$ sending $\xi$ to $c \otimes \xi$ is injective for all $e \gg 0$. Because this holds for all $c$ not in any minimal prime, we conclude that no $\xi$ can be in the tight closure of zero in $E$. The converse argument is equally easy.

\section{The Ring of Frobenius operators of an $R$-Module}

In this section, we introduce the $\operatorname{ring} \mathcal{F}(M)$ of Frobenius operators on an $R$ module $M$. The ring $\mathcal{F}(M)$ will be a certain non-commutative $\mathbb{N}$-graded ring that contains $\operatorname{End}_{R} M$ as the degree zero subring.

Because $M$ is an $R$-module, there is a natural ring map $R \rightarrow \operatorname{End}_{\mathbb{Z}}(M)$ sending $r \in R$ to the self map of $M$ given by multiplication by $r$. The kernel of this map is the annihilator of $M$. Using this natural map, we identify the $\operatorname{ring} R$ with a natural subring of $\operatorname{End}_{\mathbb{Z}}(R)$.

Consider the (iterated) Frobenius map $R \stackrel{F^{e}}{\longrightarrow} R$ sending an element $r$ to its $p^{e}$-th power $r^{p^{e}}$. Let $R\left\{F^{e}\right\}$ be the (non-commutative) associative ring extension of $R$ generated by one variable $\chi$ subject to the relations $\chi r=r^{p^{e}} \chi$ for all $r \in R$. The ring $R\left\{F^{e}\right\}$ acts on $R$ via the ring homomorphism $R\left\{F^{e}\right\} \rightarrow \operatorname{End}_{\mathbb{Z}} R$ that sends $R$ to $\operatorname{End}_{\mathbb{Z}} R$ as in the preceding paragraph and sends $\chi$ to $F^{e}$. By a slight abuse of notation we often denote $\chi$ by $F^{e}$ if this does not lead to confusion.

Because $R \subset R\left\{F^{e}\right\}$, every $R\left\{F^{e}\right\}$-module is an $R$-module. Conversely, an $R\left\{F^{e}\right\}$-module is simply an $R$-module $M$ together with an action of $F^{e}$ on $M$ that respects the above relations. Thus, to define an $R\left\{F^{e}\right\}$-module structure on an $R$-module $M$, one need only define an additive map

$$
M \stackrel{\phi_{e}}{\longrightarrow} M
$$


satisfying

$$
\phi_{e}(r m)=r^{p^{e}} \phi_{e}(m)
$$

for every $r \in R$, and every $m \in M$. If we regard the second copy of $M$ as an $R$-module via restriction of scalars from the Frobenius map $R \stackrel{F^{e}}{\longrightarrow} R$, and denote this $R$-module by ${ }^{e} M$, then we see that $\phi_{e}$ is simply an $R$-module map $M \stackrel{\phi_{e}}{\longrightarrow} M$.

Let $\mathcal{F}^{e}(M)$ be the set of all $R\left\{F^{e}\right\}$-module structures on $M$. This naturally forms an abelian group, since if two maps $M \stackrel{\phi_{e}}{\longrightarrow} M$ and $M \stackrel{\phi_{e}^{\prime}}{\longrightarrow} M$ satisfy (3.1), then so does their sum. This abelian group has a natural (left) $R$-module structure given by composition of operators: $r \in R$ acts on $\phi \in \mathcal{F}^{e}(R)$ by the composition $r \circ \phi_{e}$,

$$
M \stackrel{\phi_{e}}{\longrightarrow} M \stackrel{\text { mult by } r}{\longrightarrow} M .
$$

Thus $\mathcal{F}^{e}(M)$ forms an $R$-module in a natural way. Of course, there is also a natural right $R$-module structure given by composition in the other order, making $\mathcal{F}^{e}(M)$ an $R$-bimodule, but we will not make use of this structure. If $e=0$, then $\mathcal{F}^{e}(M)$ is simply the set $\operatorname{End}_{R}(M)$ of $R$-module endomorphisms on $M$.

3.2. Remark. The $R$-module $\mathcal{F}^{e}(M)$ of all $R\left\{F^{e}\right\}$-module structures on $M$ can be identified with the $R$-module $\operatorname{Hom}_{R}\left(R^{(e)} \otimes_{R} M, M\right)$, where here (and always, except where otherwise indicated) the homomorphisms considered are left $R$-module homomorphisms. This follows from the natural $R$-module isomorphism

$$
\begin{aligned}
\mathcal{F}^{e}(M) & \rightarrow \operatorname{Hom}_{R}\left(R^{(e)} \otimes_{R} M, M\right), \\
\left(M \stackrel{\phi_{e}}{\longrightarrow} M\right) & \mapsto\left(r \otimes m \mapsto r \phi_{e}(m)\right),
\end{aligned}
$$

whose inverse is given by

$$
\left(R^{(e)} \otimes_{R} M \stackrel{\psi}{\longrightarrow} M\right) \mapsto(m \mapsto \psi(1 \otimes m)) .
$$

This isomorphism is nothing more than the usual adjointness of extension and restriction of scalars for the Frobenius map.

We now record some useful facts about modules of Frobenius structures for later use.

3.3. Proposition. If $M$ is a Noetherian $R$-module, then the $R$-module $\mathcal{F}^{e}(M)$ of $R\left\{F^{e}\right\}$-module structures on $M$ is finitely generated over $R$. If $M$ is an Artinian module over a local ring $R$, then $\mathcal{F}^{e}(M)$ is the same whether considered as a module over $R$ or over $\hat{R}$, and $\mathcal{F}^{e}(M)$ is finitely generated over $\hat{R}$.

Proof. If $M$ is Noetherian (respectively, Artinian), then $R^{(e)} \otimes_{R} M$ is (left) Noetherian (respectively, Artinian) as well. But the $R$-module of homomorphisms between two Noetherian (respectively, two Artinian) $R$-modules is finitely generated over $R$ (respectively, over $\hat{R}$ ). Furthermore, if $M$ is Artinian over a local $\operatorname{ring}$, then $M$ is a module over both $R$ and $\hat{R}$, and a map $M \stackrel{\phi_{e}}{\longrightarrow} M$ defines an $\mathcal{F}^{e}(M)$-structure for the $R$-module $M$ if and only if it defines an $\mathcal{F}^{e}(M)$-structure for the $\hat{R}$-module $M$.

We conclude by proving the above claim that if $R$ is local and $M$ is Artinian, then $R^{(e)} \otimes_{R} M$ is Artinian. We have that $R^{(e)} \otimes_{R} M \cong \hat{R}^{(e)} \otimes_{\hat{R}} M$. Since $\hat{R}$ is complete, there exists a surjective ring homomorphism $A \rightarrow \hat{R}$ with $A$ a complete regular local ring, so every $\hat{R}$-module becomes an $A$-module via this homomorphism 
and, in the category of $\hat{R}$-modules, tensoring over $\hat{R}$ is the same as tensoring over $A$; in particular, $\hat{R}^{(e)} \otimes_{\hat{R}} M \cong \hat{R}^{(e)} \otimes_{A} M$. The induced surjection $A^{(e)} \rightarrow \hat{R}^{(e)}$ of $A$-bimodules yields a surjection $A^{(e)} \otimes_{A} M \rightarrow \hat{R}^{(e)} \otimes_{A} M$, so it is enough to show that $A^{(e)} \otimes_{A} M$ is Artinian. But $A^{(e)} \otimes_{A} M$ is supported at $m$, the maximal ideal of $A$. The functor $A^{(e)} \otimes_{A}(-)$ is exact because $A$ is regular, so the annihilator of $m^{\left[p^{e}\right]}$ in $A^{(e)} \otimes_{A} M$ is $A^{(e)} \otimes_{A}(\operatorname{socle}(M))$. But socle $(M)$ is finitely generated, hence so is $A^{(e)} \otimes_{A}(\operatorname{socle}(M))$. The socle of $A^{(e)} \otimes_{A} M$ is a submodule of $A^{(e)} \otimes_{A}(\operatorname{socle}(M))$, hence finite. Thus $A^{(e)} \otimes_{A} M$ is Artinian.

3.4. Proposition. Let $R \rightarrow S$ be a ring map that is either surjective or a localization map, and let $M$ be an $S$-module. Then the module of Frobenius structures $\mathcal{F}^{e}(M)$ is independent of whether we view $M$ as an $R$-module or an $S$-module.

Proof. An additive map $M \stackrel{\phi_{e}}{\longrightarrow} M$ defines an $R\left\{F^{e}\right\}$-module structure on $M$ if and only if it defines an $S\left\{F^{e}\right\}$-module structure on $M$, because for each $x$ in $M$, $\phi_{e}(r x)=r^{p^{e}} \phi_{e}(x)$ holds for all $r \in R$ if and only if it holds for all $r \in S$.

Given an $R\left\{F^{e}\right\}$-structure $\phi_{e}$ and an $R\left\{F^{e^{\prime}}\right\}$-structure $\phi_{e^{\prime}}$ on $M$, it is easy to check that their composition $\phi_{e} \circ \phi_{e^{\prime}}$ is an $R\left\{F^{e+e^{\prime}}\right\}$-structure on $M$.

3.5. Definition. The ring of Frobenius operators on $M$ is the graded associative (not necessarily commutative) ring $\mathcal{F}(M)=\mathcal{F}^{0}(M) \oplus \mathcal{F}^{1}(M) \oplus \mathcal{F}^{2}(M) \oplus \ldots$

The ring $\mathcal{F}(M)$ contains $\mathcal{F}^{0}(M)=\operatorname{End}_{R}(M)$ as a subring which in turn is an $R$-algebra in a natural way. If $M$ is Noetherian or if $R$ is complete local and $M$ is Artinian, each summand $\mathcal{F}^{e}(M)$ is finitely generated over $R$ and hence over $\mathcal{F}^{0}(M)$; however, we do not know if $\mathcal{F}(M)$ is finitely generated as a ring extension of $\mathcal{F}^{0}(M)$ in general, or not.

The ring $\mathcal{F}(M)$ acts on $M$ via the ring homomorphism $\mathcal{F}(M) \rightarrow \operatorname{End}_{\mathbb{Z}} M$ that sends every $\phi_{e} \in \mathcal{F}^{e}(M) \subset \mathcal{F}(M)$ to the same element $\phi_{e} \in \mathcal{F}^{e}(M) \subset \operatorname{End}_{\mathbb{Z}} M$.

3.6. Example. The ring of Frobenius operators on $R$ is the ring $R\{F\}$ discussed at the beginning of the section. Indeed, for each $e$, the $R$-module $\mathcal{F}^{e}(R)$ is the free (left) $R$-submodule of $\operatorname{End}_{\mathbb{Z}}(R)$ generated by the $e$-th iterate of the Frobenius map $R \stackrel{F^{e}}{\longrightarrow} R$. To see this, note that any Frobenius structure $R \stackrel{\phi_{e}}{\longrightarrow} R$ is determined by the image of $1 \in R$ since $\phi_{e}(r)=r^{p^{e}} \phi_{e}(1)$; furthermore, $\phi_{e}(1)=1$ if and only if $\phi_{e}=F^{e}$. Setting $\phi_{e}(1)=r$, we see that each element of $\mathcal{F}^{e}(R)$ can be written as $r F^{e}$, where $r$ is uniquely determined, proving that $\mathcal{F}^{e}(R)=R F^{e}$. It follows from (3.1) that $\phi_{e} r=r^{p^{e}} \phi_{e}$ for every $\phi_{e} \in \mathcal{F}(M)$ and $r \in R$, so $\mathcal{F}(R)$ is the ring extension of $R$ generated by one variable $F$ subject to the relations $F r=r^{p^{e}} F$ for all $r \in R$, which is to say, it is $R\{F\}$.

3.7. Example. If $(R, m)$ is a complete local ring of positive dimension $d$ which satisfies Serre's $S_{2}$ condition, then the ring of Frobenius structures $\mathcal{F}\left(H_{m}^{d}(R)\right)$ on the local cohomology module $H_{m}^{d}(R)$ is isomorphic to $R\{F\}$. Indeed, the natural map

$$
R\{F\} \rightarrow \mathcal{F}\left(H_{m}^{d}(R)\right)
$$

sending elements of $R$ to their usual action by multiplication on $H_{m}^{d}(R)$ and sending $F$ to the natural action on $H_{m}^{d}(R)$ (induced by the Frobenius $R \stackrel{F}{\longrightarrow} R$ via functoriality of local cohomology) is an isomorphism. To see this, represent $H_{m}^{d}(R)$ as the 
quotient module

$$
\frac{R_{x_{1} \ldots x_{d}}}{\sum_{i} R_{x_{1} \ldots x_{i-1} x_{i+1} \ldots x_{d}}}
$$

where $x_{1}, \ldots, x_{d}$ is a system of parameters of $R$. In this notation, the natural action of the Frobenius $F^{e}$ on $H_{m}^{d}(R)$ looks like

$$
\frac{r}{\left(x_{1} \ldots x_{d}\right)^{s}} \mapsto \frac{r^{p^{e}}}{\left(x_{1} \ldots x_{d}\right)^{s p^{e}}}
$$

on coset representatives $\frac{r}{\left(x_{1} \ldots x_{d}\right)^{s}}$, and it is easy to check that it defines a Frobenius structure on $H_{m}^{d}(R)$. The corresponding $R$-module map (see Remark 3.2)

$$
\begin{aligned}
R^{(e)} \otimes_{R} H_{m}^{d}(R) & \rightarrow H_{m}^{d}(R), \\
r \otimes x & \mapsto r F^{e}(x),
\end{aligned}
$$

is an isomorphism. This is easy to verify after noting that the maps

$$
R^{(e)} \otimes R_{x} \stackrel{r^{\prime} \otimes r / x^{s} \mapsto r^{\prime}\left(r / x^{s}\right)^{p^{e}}}{\longrightarrow} R_{x}
$$

are isomorphisms for every $x$ and that tensoring preserves cokernels. Thus

$$
\mathcal{F}^{e}\left(H_{m}^{d}(R)\right)=\operatorname{Hom}_{R}\left(R^{(e)} \otimes H_{m}^{d}(R), H_{m}^{d}(R)\right) \cong \operatorname{Hom}_{R}\left(H_{m}^{d}(R), H_{m}^{d}(R)\right) \cong R .
$$

It is this last isomorphism where the $S_{2}$ (and of course completeness) assumption is used. So $\mathcal{F}^{e}\left(H_{m}^{d}(R)\right)$ is generated as an $R$-module by any invertible element (isomorphism) of $\operatorname{Hom}_{R}\left(R^{(e)} \otimes H_{m}^{d}(R), H_{m}^{d}(R)\right)$, and so in particular by $F^{e}$. Finally, since $F^{e} \circ F^{e^{\prime}}=F^{e+e^{\prime}}$, the natural map $R\{F\} \rightarrow \mathcal{F}\left(H_{m}^{d}(R)\right)$ above is an isomorphism.

3.8. Example. The isomorphism in Example 3.7 can be generalized to an arbitrary local ring of dimension $d$ as follows. Let $S$ denote the $S_{2}$-ification of the completion $\hat{R}$ of $R$ (this construction is reviewed below). Then the ring of Frobenius structures $\mathcal{F}\left(H_{m}^{d}(R)\right)$ on the local cohomology module $H_{m}^{d}(R)$ is isomorphic to $S\{F\}$.

To see this, first recall that the $S_{2}$-ification is defined for any $\operatorname{ring} T$ admitting a canonical module $\omega_{T}$. Namely, the $T$-module $T^{\prime}=\operatorname{Hom}_{T}\left(\omega_{T}, \omega_{T}\right)$ admits the structure of a commutative ring, and the natural map $T \rightarrow T^{\prime}$ sending elements of $T$ to their action on $\omega_{T}$ by multiplication is a finite map to an $S_{2}$ ring. The map $T \rightarrow T^{\prime}$ is an isomorphism if and only if $T$ is $S_{2}$ and equidimensional (the latter follows from $S_{2}$-ness if $T$ is catenary), and it is injective if and only if $T$ is equidimensional and unmixed. All these facts are nicely summarized in the form we use them in [HH5], with original proofs due to Aoyama A1] [A2].

Now, by Proposition 3.3, the ring $\mathcal{F}\left(H_{m}^{d}(R)\right)$ is the same whether considered over $R$ or over $\hat{R}$, so we might as well assume that $R$ is complete. In this case, using the adjointness of tensor and hom, one easily checks that $S=\operatorname{Hom}_{R}\left(\omega_{R}, \omega_{R}\right) \cong$ $\operatorname{Hom}_{R}\left(H_{m}^{d}(R), H_{m}^{d}(R)\right)$. So there is a natural map

$$
S\{F\} \rightarrow \mathcal{F}\left(H_{m}^{d}(R)\right)
$$

generalizing the map above in Example 3.7, and the argument above shows that it is an isomorphism.

3.9. Remark. The functor $H_{I}^{i}(-)$, for any ideal $I \subset R$ and any $i$, induces (by restriction) a functor from the category of $R\{F\}$-modules to the category of $R\{F\}$ modules. Indeed, since $H_{I}^{i}(M)$ can be computed as the cohomology of a complex 
consisting of localizations of $M$ with maps (essentially) the natural localization maps, and because the action of $R\{F\}$ on $M$ passes uniquely to any localization of $M$, there is a natural induced action of $R\{F\}$ on $H_{I}^{i}(M)$ which is functorial in $M$.

Finally, we point out that the $\mathcal{F}$-modules we are most interested in, namely the top local cohomology module and the injective hull of the residue field of a local ring, are finitely generated.

3.10. Proposition. Let $(R, m)$ be a local ring of positive dimension $d$.

(1) The local cohomology module $H_{m}^{d}(R)$ is a cyclic $R\{F\}$ module, and hence a cyclic $\mathcal{F}\left(H_{m}^{d}(R)\right)$-module.

(2) If $\hat{R}$ is unmixed and generically Gorenstein (for example, if $R$ is excellent and reduced), then $E$ is a finitely generated $\mathcal{F}(E)$-module. More precisely, let $D$ be the set of integers such that $\delta \in D$ if and only if there is a minimal prime of $\hat{R}$ of dimension $\delta$. Then $E$ is generated as $\mathcal{F}(E)$-module by card $D$ elements.

Proof. In the notation of the preceding examples we claim that $H_{m}^{d}(R)$ is a cyclic $R\{F\}$-module generated by the image of $\frac{1}{x_{1} \ldots x_{d}}$ in $H_{m}^{d}(R)$. Indeed, if $\eta \in H_{m}^{d}(R)$ is represented by $\frac{r}{\left(x_{1} \ldots x_{d}\right)^{s}}$, then for $p^{e} \geq s$ we can write

$$
\eta=\frac{r}{\left(x_{1} \ldots x_{d}\right)^{s}}=\frac{r\left(x_{1} \ldots x_{d}\right)^{p^{e}-s}}{\left(x_{1} \ldots x_{d}\right)^{p^{e}}}=r\left(x_{1} \ldots x_{d}\right)^{p^{e}-s} F^{e}\left(\frac{1}{x_{1} \ldots x_{d}}\right) .
$$

Since $R\{F\}$ maps to $\mathcal{F}\left(H_{m}^{d}(R)\right) \cong S\{F\}$ (via the map induced by the natural map $R \rightarrow S$ ), any cyclic generator for $H_{m}^{d}(R)$ as an $R\{F\}$-module will also generate it as an $\mathcal{F}\left(H_{m}^{d}(R)\right)$-module. This proves (1).

To prove (2), we may assume that $R$ is complete, since, as we have seen in Proposition 3.3, the $\mathcal{F}(E)$-module structure of $E$ is the same whether considered over $R$ or over $\hat{R}$.

First we consider the case where $R$ is equidimensional. Since $\operatorname{card} D=1$, we need to prove that $E$ is cyclic. Because $R$ is complete, it admits a canonical module $\omega$, and because $R$ is generically Gorenstein, $\omega_{P}=R_{P}$ for every minimal prime of $R$. Thus there is a map $\omega \rightarrow R$ whose kernel and cokernel have support of dimension strictly less than $d$. Let $\omega^{\prime}$ be the image of this homomorphism.

Because $H_{m}^{i}(M)$ vanishes whenever $i$ exceeds the dimension of $M$, this homomorphism induces an isomorphism $H_{m}^{d}(\omega) \cong H_{m}^{d}\left(\omega^{\prime}\right)$ and a surjection $H_{m}^{d}\left(\omega^{\prime}\right) \rightarrow$ $H_{m}^{d}(R)$. Because the usual Frobenius structure $R \stackrel{r \mapsto r^{p^{e}}}{\longrightarrow} R$ on $R$ sends every ideal of $R$ to itself, it induces a Frobenius structure on every ideal and in particular on $\omega^{\prime}$. Thus the surjection $H_{m}^{d}(\omega) \rightarrow H_{m}^{d}(R)$ is a homomorphism of $R\{F\}$ modules. Now if $R$ were $S_{2}$, we would have a natural isomorphism $H_{m}^{d}(\omega) \cong E$, and hence a surjection $E \rightarrow H_{m}^{d}(R)$ of $R\{F\}$-modules. More generally, we have an isomorphism $H_{m}^{d}(\omega) \cong S^{\prime}$, and hence a surjection $S^{\prime} \rightarrow H_{m}^{d}(R)$ of $R\{F\}$ modules, where $S^{\prime}=\operatorname{Hom}_{R}(S, E)$ is the Matlis dual of $\mathrm{S}$ in the category of $R$ modules. Indeed, by local duality, $H_{m}^{d}(\omega)$ is Matlis dual to $\operatorname{Hom}_{R}(\omega, \omega)=S$, so $H_{m}^{d}(\omega) \cong \operatorname{Hom}_{R}(S, E) \cong S^{\prime}$.

Let $\eta \in H_{m}^{d}(R)$ be a cyclic $R\{F\}$-module generator of $H_{m}^{d}(R)$ (as proved in (1)) and let $\xi \in S^{\prime}$ be a preimage of $\eta$. We claim that $S^{\prime}$ is generated as an $R\{F\}$-module by $\xi$. Otherwise, let $M$ be the proper submodule of $S^{\prime}$ generated by $\xi$. Because $M$ surjects onto $H_{m}^{d}(R)$, it must have zero annihilator: our assumption that $R$ is 
equidimensional and unmixed ensures that the annihilator of $H_{m}^{d}(R)$ (which equals the annihilator of its Matlis dual, $\omega$ ) is zero [HH5, 2.2e]. Note that if $R$ had been $S_{2}$, the proof would be complete (because then $S^{\prime} \cong E$ and the only submodule of $E$ with zero annihilator is $E$ itself), but some work remains in general. Applying Matlis duality to the proper inclusion of $R$-modules $M \subset S^{\prime}$, we get a surjection $S \rightarrow M^{\prime}=\operatorname{Hom}_{R}(M, E)$. Because the map $R \rightarrow S$ is an isomorphism at minimal primes and the annihilator of $M^{\prime}$ (which equals the annihilator of $M$ ) is zero, this surjection becomes an isomorphism after localization at minimal primes. Thus, the kernel is annihilated by some element of $R$ not in any minimal prime of $R$. This contradicts the $S_{2}$ property of the $R$-module $S$, so we have proved that $S^{\prime}$ is generated as an $R\{F\}$ module by $\xi$.

The inclusion $R \rightarrow S$ leads, via Matlis duality over $R$, to a surjection $S^{\prime} \rightarrow E$. Let $a \in R$ be any element of $R$, not in a minimal prime of $R$, that kills the module $S / R$. The element $a$ annihilates the kernel $K$ of $S^{\prime} \rightarrow E$, and thus the element $G=a^{p} F=F a \in R\{F\}$ acts on $E$ and the map $S^{\prime} \rightarrow E$ is a map of $R\{G\}$-modules. Here, and in the rest of the proof, $R\{G\}$, where $G \in \mathcal{F}^{1}(E)$, is the subring of $\mathcal{F}(E)$ generated by $R=\mathcal{F}^{0}(E)$ and $G$. It is enough to show that $S^{\prime}$ is a cyclic $R\{G\}$ module, for the image of a cyclic generator will generate $E$ as an $R\{G\}$-module, and hence as an $\mathcal{F}(E)$-module.

To this end, note that because $S$ is $S_{2}$, multiplication by $a^{p}$ on $S$ is injective, and hence multiplication by $a^{p}$ on its dual $S^{\prime}$ is surjective. Thus we can find an element $\nu \in S^{\prime}$ such that $a^{p} \nu=\xi$, our cyclic generator for $S^{\prime}$ as an $R\{F\}$ module. Now repeatedly using the relation $F r=r^{p} F$ for all $r \in R$, we see that $G^{t}(\nu)=(F a)^{t}(\nu)=a^{p+\cdots+p^{t}} F^{t}(\nu)$. Multiplying by $a^{b}$ where $b=p^{t+1}-\left(p+\cdots+p^{t}\right)$, we get $a^{b} G^{t}(\nu)=a^{p^{t+1}} F^{t}(\nu)=\left(a^{p}\right)^{p^{t}} F^{t}(\nu)=F^{t}\left(a^{p} \nu\right)=F^{t}(\xi)$. Thus the $R\{G\}$ submodule generated by $\nu$ contains the $R\{F\}$-submodule generated by $\xi$, which we have verified already is all of $S^{\prime}$. This completes the proof in the case where $R$ is equidimensional.

In the general case, let $I_{t}$ be the intersection of the primary components (in the primary decomposition of the zero ideal) corresponding to the minimal primes of dimension $t$, and let $R_{t}=R / I_{t}$. Let $E_{t}=\operatorname{Hom}_{R}\left(R_{t}, E\right) \cong\left\{e \in E \mid I_{t} e=0\right\} \subset E$. Thus $E_{t}$ is isomorphic to the injective hull of the residue field of $R_{t}$ in the category of $R_{t}$-modules. The intersection of the kernels of $R \rightarrow R_{t}$, over all $t$, is zero; hence the sum of $E_{t}$, over all $t$, is $E$. Thus it is enough to prove that for each $t$ there exists an element $G_{t} \in \mathcal{F}^{1}(E)$ such that $G_{t}$ sends $E_{t}$ to $E_{t}$ making $E_{t}$ a cyclic $R\left\{G_{t}\right\}$-module, for then the (finite) set of $\nu_{t}$, over all $t$, would generate $E$ as an $\mathcal{F}(E)$-module, where $\nu_{t}$ generates $E_{t}$ as an $R\left\{G_{t}\right\}$-module.

We have an exact sequence $0 \rightarrow I_{t} \rightarrow R \rightarrow R_{t} \rightarrow 0$. Applying Matlis duality we get an exact sequence $0 \rightarrow E_{t} \rightarrow E \rightarrow I_{t}^{\prime} \rightarrow 0$. Applying the Frobenius we get an exact sequence $\operatorname{Tor}_{1}^{R}\left(R^{(1)}, I_{t}^{\prime}\right) \rightarrow R^{(1)} \otimes_{R} E_{t} \rightarrow R^{(1)} \otimes_{R} E \rightarrow R^{(1)} \otimes_{R} I_{t}^{\prime} \rightarrow 0$. Let $J_{t}$ be the intersection of all $I_{i}$ with $i \neq t$. Then $J_{t} I_{t}=0$. Let $b \in J_{t}$ be an element not in any minimal prime of $I_{t}$. Then $b$ annihilates $I_{t}$ and hence $I_{t}^{\prime}$ and $\operatorname{Tor}_{1}^{R}\left(R^{(1)}, I_{t}^{\prime}\right)$. Taking the Matlis dual of the above exact sequence, we get an exact sequence $\mathcal{F}^{1}(E)=\left(R^{(1)} \otimes_{R} E\right)^{\prime} \rightarrow\left(R^{(1)} \otimes_{R} E_{t}\right)^{\prime} \rightarrow \operatorname{Tor}_{1}^{R}\left(R^{(1)}, I_{t}^{\prime}\right)^{\prime}$, where the module on the right is annihilated by $b$. Thus the cokernel of the natural map $\mathcal{F}^{1}(E) \rightarrow\left(R^{(1)} \otimes_{R} E_{t}\right)^{\prime}$ is annihilated by $b$.

Applying $\operatorname{Hom}_{R}\left(R^{(1)} \otimes_{R} E_{t},-\right)$ to the exact sequence $0 \rightarrow E_{t} \rightarrow E$, we get an exact sequence $0 \rightarrow \mathcal{F}^{1}\left(E_{t}\right) \rightarrow\left(R^{(1)} \otimes_{R} E_{t}\right)^{\prime}$, i.e. $\mathcal{F}^{1}\left(E_{t}\right)$ is a submodule of 
$\left(R^{(1)} \otimes_{R} E_{t}\right)^{\prime}$. Hence $b \mathcal{F}^{1}\left(E_{t}\right)$ is in the image of the last map from the preceding paragraph. That is, if $\tilde{G} \in \mathcal{F}^{1}\left(E_{t}\right)$, then $b \tilde{G}$ is induced by some $G \in \mathcal{F}^{1}(E)$.

Let $\tilde{G}_{t} \in \mathcal{F}^{1}\left(E_{t}\right)$ be such that $E_{t}$ is a cyclic $R_{t}\left\{\tilde{G}_{t}\right\}$-module. That such an element $\tilde{G}_{t}$ exists, was proven in the course of our proof of the equidimensional case above. The same argument as in the last paragraph of our proof of the equidimensional case shows that $E_{t}$ is a cyclic $R_{t}\left\{b \tilde{G}_{t}\right\}$-module. But the action of $b \tilde{G}_{t}$ on $E_{t}$ is induced by some $G_{t} \in \mathcal{F}^{1}(E)$, so $E_{t}$ is a cyclic $R\left\{G_{t}\right\}$-module.

\section{A characterization of Strong F-Regularity IN TERMS OF FROBENIUS OPERATORS}

The goal of this section is to prove the following characterization of strongly F-regular rings. As before, $E$ denotes the direct sum $\bigoplus E(R / m)$ over all maximal ideals $m$ in $R$ of the injective hulls of the simple $R$-modules $R / m$.

4.1. Theorem. Let $R$ be a reduced F-finite ring. Then $R$ is strongly F-regular if and only if $E$ is a semi-simple $\mathcal{F}(E)$ module. If $R$ is local, it is strongly F-regular if and only if $E$ is simple as an $\mathcal{F}(E)$-module.

For Gorenstein rings $R$, this follows from [S4, Theorem 2.6]. Theorem 4.1 is important in that it finally frees this sort of tight closure argument from the usual dependence on some sort of "nearly Gorenstein" hypothesis. By adopting the convention for defining strongly F-regular rings in the non-F-finite case described in 2.8 , the theorem holds for reasonable rings even without the assumption that $R$ is F-finite; see Remark 6.4.

Before proving this theorem, we observe that tight closure modules provide a multitude of examples of non-trivial $\mathcal{F}$-modules.

4.2. Proposition. Let $M$ be any $R$-module. Then the modules $0_{M}^{*}$ and $0_{M}^{* f g}$ are $\mathcal{F}(M)$-submodules of $M$.

Proof. First we prove that $0_{M}^{*}$ is an $\mathcal{F}(M)$-submodule. Let $x \in 0_{M}^{*}$. Choose $c \in R$ not in any minimal prime of $R$ such that $c \otimes x$ is zero in $R^{(e)} \otimes_{R} M$ for every $e \gg 0$. We need to show that $\phi_{e^{\prime}}(x) \in 0_{M}^{*}$ for every $\phi_{e^{\prime}} \in \mathcal{F}^{e^{\prime}}(M)$. Set $\phi_{e^{\prime}}(x)=y$. Let $R^{\left(e^{\prime}\right)} \otimes_{R} M \stackrel{\psi}{\longrightarrow} M$ correspond to $\phi_{e^{\prime}}$ (as in Remark 3.2), so that $y=\psi(1 \otimes x)$. Then $c \otimes y \in R^{(e)} \otimes_{R} M$ is the image of $c \otimes x$ under the map $R^{\left(e+e^{\prime}\right)} \otimes_{R} M \cong R^{(e)} \otimes_{R} R^{\left(e^{\prime}\right)} \otimes_{R} M \stackrel{\operatorname{id} \otimes \psi}{\longrightarrow} R^{(e)} \otimes_{R} M$. But $c \otimes x=0$ for large $e ;$ hence $c \otimes y=0$ in $R^{(e)} \otimes M$ for $e \gg 0$. We conclude that $y \in 0_{M}^{*}$.

Next we show that $0_{M}^{* f g}$ is an $\mathcal{F}(M)$-submodule. Let $N \stackrel{\imath}{\hookrightarrow} M$ be the inclusion of a finitely generated submodule $N$ in $M$. Suppose that $x \in 0_{N}^{*}$, and let $c \in R$ not in any minimal prime of $R$ be such that $c \otimes x=0$ in $R^{(e)} \otimes_{R} N$ for all $e \gg 0$. Let $\phi_{e^{\prime}}, y$ and $\psi$ be as above. Let $N^{\prime} \subset M$ be the image of the composition $R^{\left(e^{\prime}\right)} \otimes_{R} N \stackrel{\text { id } \otimes \imath}{\longrightarrow} R^{\left(e^{\prime}\right)} \otimes_{R} M \stackrel{\psi}{\longrightarrow} M$. Since $N$ is finitely generated, so is $R^{\left(e^{\prime}\right)} \otimes_{R} N$, and hence so is its image $N^{\prime}$. Thus it is enough to prove that $y \in 0_{N^{\prime}}^{*}$. This follows from the fact that $c \otimes y \in R^{(e)} \otimes_{R} N^{\prime}$ is the image of $c \otimes x \in R^{\left(e+e^{\prime}\right)} \otimes_{R} N$ in a similar way as in the previous paragraph.

4.3. Example. For example, the modules $0_{E}^{*}, 0_{E}^{* f g}$ and $0_{H_{m}^{d}(R)}^{*}$ are $\mathcal{F}$-submodules of $E, E$, and $H_{m}^{d}(R)$ respectively.

\footnotetext{
${ }^{1}$ By semi-simple, we mean a (possibly infinite) direct sum of simple modules.
} 
We are especially interested in studying the $\mathcal{F}(E)$ submodule $0_{E}^{*}$ of $E$. The following theorem describes this tight closure module for an arbitrary local ring.

4.4. Theorem. Let $R$ be a local ring, and let $E$ denote an injective hull of its residue field. Then the tight closure of zero in $E$ is the sum of all $\mathcal{F}(E)$-submodules of $E$ whose annihilators have positive height.

Proof. Let $\mathcal{M}$ be the sum of all $\mathcal{F}(E)$-submodules of $E$ whose annihilators have positive height, that is, whose annihilators are not contained in any minimal prime.

To see that $0_{E}^{*} \subset \mathcal{M}$, consider any $z \in 0_{E}^{*}$. By definition, there exists $c$ not contained in any minimal prime of $R$ such that $c \otimes z$ is zero in $R^{(e)} \otimes_{R} E$ for all $e \gg 0$. The image of $c \otimes z$ in $E$ via any Frobenius structure $\phi_{e}$ on $E$ (interpreted as a map $\left.R^{(e)} \otimes_{R} E \rightarrow E\right)$ is of course also zero. In particular, $c$ kills every $\phi_{e}(z)$; hence $c$ kills the cyclic $\mathcal{F}(E)$-submodule of $E$ generated by $z$. This means that $z$ generates a proper $\mathcal{F}(E)$-submodule of $E$ whose annihilator contains $c$ and is therefore not contained in any minimal prime of $R$. In particular, $z \in \mathcal{M}$, so that $0_{E}^{*} \subset \mathcal{M}$.

For the reverse inclusion, first note that since the annihilator of the sum of two modules is the intersection of their individual annihilators, the set of $\mathcal{F}(E)$ submodules of $E$ with positive-height annihilators is closed under (finite) sums. Thus if $z \in \mathcal{M}$, then the annihilator of the $\mathcal{F}(E)$-submodule generated by $z$ has positive height. Let $M$ denote this $\mathcal{F}(E)$ module, and let $c$ be an element not in any minimal prime that kills $M$. Let $N$ be the $R$-submodule of $R^{(e)} \otimes_{R} E$ generated by $1 \otimes z$. Because $N \subset R^{(e)} \otimes_{R} E$, we have a surjection of the Matlis duals $\operatorname{Hom}_{R}\left(R^{(e)} \otimes_{R} E, E\right) \rightarrow \operatorname{Hom}_{R}(N, E)$. Since $M$ is an $\mathcal{F}(E)$-submodule, for each $\phi \in \operatorname{Hom}_{R}\left(R^{(e)} \otimes_{R} E, E\right)$ we have $\phi(1 \otimes z) \in M$. This means that the image $\bar{\phi}$ of $\phi$ in $\operatorname{Hom}_{R}(N, E)$ has the property that $\bar{\phi}(N) \subset M$. Because $c$ kills $M$, we conclude that $c$ kills every $\bar{\phi} \in \operatorname{Hom}_{R}(N, E)$. But a Noetherian module and its Matlis dual have the same annihilator, so $c$ kills $N$. It follows that $c$ kills $1 \otimes z$ in every $R^{(e)} \otimes_{R} E$, so that $z$ is in $0_{E}^{*}$.

We will eventually show that under mild conditions on a local ring $R$, the module $0_{E}^{*}$ is the intersection of the maximal proper $\mathcal{F}(E)$-submodules of $E$. In this section, we treat only the key case of a complete local domain, where the ideas are more transparent. A lemma is needed.

4.5. Lemma. Assume that $(R, m)$ is unmixed and analytically generically Gorenstein, that is, that $\hat{R}$ becomes a Gorenstein ring after localization at any minimal prime (for example, $R$ could be a complete local domain). Then every proper $\mathcal{F}(E)$ submodule of $E$ is contained in a maximal proper $\mathcal{F}(E)$-submodule of $E$.

Proof. This follows from Zorn's lemma. If $M \subset M_{1} \subset M_{2} \subset M_{3} \subset \ldots$ is an increasing chain of proper $\mathcal{F}(E)$-submodules of $E$ containing $M$, then we only need to show that their union is also a proper $\mathcal{F}(E)$-submodule of $E$. But if not, the union must contain the finite set of generators for $E$ guaranteed by Proposition 3.10. But since this set of generators is finite, it is contained in some $M_{i}$, and $M_{i}$ is not proper.

Note that even if $R$ does not satisfy the mild hypothesis of Lemma 4.5, the proof is still valid whenever $E$ is a finitely generated $\mathcal{F}(E)$-module. It is reasonable to expect that $E$ is always a finitely generated $\mathcal{F}(E)$-module, but we have not been able to prove this in general (see Proposition 3.10). 
4.6. Corollary. Let $(R, m)$ be analytically reduced and irreducible (that is, assume that $\hat{R}$ is a domain). Then $E$ has a unique maximal proper $\mathcal{F}(E)$-submodule, and it is the tight closure of zero in E, computed over $\hat{R}$.

Caution. It is not a priori clear that the computation of $0_{E}^{*}$ is independent of whether we view the ring as $R$ or as $\hat{R}$. Although clearly $\left(0_{E}^{*}\right)_{R} \subset\left(0_{E}^{*}\right)_{\hat{R}}$ (with the subscripts indicating the ring), the reverse inclusion is not obvious, since the required ' $c$ ' in the definition of tight closure may exist in $\hat{R}$ but not in $R$. After developing more machinery in Section 6, we will indeed show that under mild hypothesis on $R, 0_{E}^{*}$ is independent of whether we perform the computation over $R$ or over $\hat{R}$. In Theorem 6.2 , we will generalize Corollary 4.6 to show that even when $\hat{R}$ is not a domain, the module $0_{E}^{*}$ is the unique proper $\mathcal{F}(E)$-submodule of $E$ maximal among those whose annihilators in $\hat{R}$ are not contained in any minimal prime.

Proof of 4.6. The $\mathcal{F}(E)$-submodules of $E$ are the same whether considered over $R$ or $\hat{R}$, so there is no loss of generality in assuming that $R$ is complete. Over a complete local ring $R$, the submodules of $E$ are in one-one correspondence with the ideals of $R$; the mutually inverse assignment as follows: $M \subset E$ corresponds to $\operatorname{Ann}_{R}(M) \subset R$ and $I \subset R$ corresponds to $\operatorname{Ann}_{E}(I) \subset E$.

By Lemma $4.5, E$ has a maximal proper $\mathcal{F}(E)$-module. If $M_{1}$ and $M_{2}$ are two distinct maximal $\mathcal{F}(E)$ submodules, then $M_{1}+M_{2}$, being an $\mathcal{F}(E)$-module strictly larger than both $M_{1}$ and $M_{2}$, cannot be proper. But its annihilator in $\hat{R}$ is the intersection $\operatorname{Ann}_{\hat{R}}\left(M_{1}\right) \cap \operatorname{Ann}_{\hat{R}}\left(M_{2}\right)$ of two non-zero ideals in the domain $\hat{R}$, and so cannot be the zero ideal. Because $E$ is faithful, we have a contradiction. So $E$ has a unique maximal $\mathcal{F}(E)$-submodule, and by Theorem 4.4 , it is $0_{E}^{*}$.

4.7. Remark. Corollary 4.6 does not really require that $R$ be analytically reduced and irreducible. The argument shows that whenever the set of $\mathcal{F}(E)$-modules whose annihilator is not contained in any minimal prime of $R$ has a unique maximal proper element, then that unique maximal element must be $0_{E}^{*}$. This conclusion holds even when $0_{E}^{*}$ is computed over $R$ instead of over $\hat{R}$. What is not so clear without passing to the complete case is that the above maximal proper $\mathcal{F}(E)$-submodule of $E$ exists. After developing more machinery we will prove this in Section 6.

Proof of Theorem 4.1. We begin by reducing to the local case.

First, the $R$-module decomposition $E=\bigoplus E(R / m)$ is also an $\mathcal{F}(E)$-module decomposition. To see this, consider one summand $E(R / m)$. We need to show that this summand is stable under the action of every $\phi_{e}$ in $\mathcal{F}^{e}(E)$. Let $\tilde{\eta}=$ $(0,0, \ldots, 0, \eta, 0,0, \ldots)$ be an element of the submodule $E(R / m)$, and suppose its image under $\phi_{e}$ is $\left(\mu_{1}, \mu_{2}, \ldots, \mu_{n}, 0,0, \ldots\right)$ in $E$. Since $\eta$ is killed by a power of $m$, so is $\phi_{e}(\tilde{\eta})$, as $\phi_{e}\left(m^{t} \tilde{\eta}\right)=\left(m^{t}\right)^{\left[p^{e}\right]} \phi_{e}(\tilde{\eta})$. But the elements of $R$ not in a maximal ideal $n$ of $R$ act invertibly on $E(R / n)$, so if $\mu_{i} \in E(R / n)$ (where $n$ is distinct from $m$ ) is killed by a power of $m$, then $\mu_{i}$ must be zero. This says that each Frobenius operator in $\mathcal{F}(E)$ preserves each summand $E(R / m)$, so that $E=\bigoplus E(R / m)$ is an $\mathcal{F}(E)$-module decomposition.

Now $E$ will be semi-simple if and only if each summand $E(R / m)$ is semi-simple. But because $E(R / m)$ is indecomposable (even as an $R$-module), this occurs if and only if each $E(R / m)$ is a simple $\mathcal{F}(E)$-module. Clearly the $\mathcal{F}(E)$-module structure of the summand $E(R / m)$ is the same as its $\mathcal{F}(E(R / m))$-module structure, and by 
Proposition 3.4, this is the same as the $\mathcal{F}\left(E_{R_{m}}\right)$-module structure of the module $E_{R_{m}}=E(R / m)$, considered over the local ring $R_{m}$. So $E$ is a semi-simple $\mathcal{F}(E)$ module if and only if for each maximal ideal $m$ of $R$, the injective hull of the residue field $E_{R_{m}}$ of the local ring $R_{m}$ is a simple $\mathcal{F}\left(E_{R_{m}}\right)$-module.

On the other hand, the ring $R$ is strongly F-regular if and only if for all maximal ideals of $R, R_{m}$ is strongly F-regular [HH2, 3.1a]. Thus we are reduced to proving the second assertion: a reduced, F-finite local ring $R$ is strongly F-regular if and only if $E_{R}$ is a simple $\mathcal{F}(E)$-module.

Now we reduce to the complete case. We have already seen that the $\mathcal{F}(E)$ module structure of $E$ is the same whether considered over $R$ or $\hat{R}$. On the other hand, an F-finite local ring $R$ is strongly F-regular if and only if its completion is strongly F-regular. Indeed, since $R \rightarrow \hat{R}$ is faithfully flat, the strong F-regularity of $\hat{R}$ immediately implies the strong F-regularity of $R$ [HH2, 3.1b]. Conversely, since $R$ is excellent, we can choose $c$ in $R$ such that both $R_{c}$ and $\hat{R}_{c}$ are regular. If $R$ is strongly F-regular, the $R$-module inclusion $R c^{1 / p^{e}} \hookrightarrow R^{1 / p^{e}}$ splits for some $e$. But then, tensoring with $\hat{R}$, the $\hat{R}$-module inclusion

$$
\hat{R} c^{1 / p^{e}} \hookrightarrow R^{1 / p^{e}} \otimes_{R} \hat{R} \cong \hat{R}^{1 / p^{e}}
$$

splits over $\hat{R}$, where the isomorphism on the right follows from the F-finiteness of $R$. This shows that $\hat{R}$ is strongly F-regular [HH2, 3.3].

Now we assume that $R$ is a complete local ring. If $R$ is strongly F-regular, then $R$ is a domain and $0_{E}^{*}=0$ by Proposition 2.9. But since $0_{E}^{*}$ is the maximal proper $\mathcal{F}(E)$-submodule of $E$, it follows that $E$ is a simple $\mathcal{F}(E)$-module. On the other hand, if $E$ is a simple $\mathcal{F}(E)$-module, then all proper $\mathcal{F}(E)$-modules, including $0_{E}^{*}$, are zero. Again by 2.9 , we conclude that $R$ is strongly F-regular.

\section{AnNihilators of $\mathcal{F}(E)$-submodules of $E$}

The results of the previous section indicate that understanding the $\mathcal{F}(E)$ submodules of $E$ is important for understanding tight closure. In this section, we develop a technique for analyzing $\mathcal{F}(E)$-modules in terms of their annihilators. A key idea is proved in Proposition 5.3: annihilators of $\mathcal{F}(E)$-modules are well-behaved under localization. This will eventually be the main point in our proof that the ideal $\tilde{\tau}$ annihilating $0_{E}^{*}$ behaves well under localization. This will also allow us to put a natural scheme structure on the locus of non-strongly F-regular points of a ring.

5.1. Notation. Throughout this section, $R$ will be assumed to be a homomorphic image of a regular local ring $A$ containing a field of characteristic $p$. We denote by $I \subset A$ the kernel of the surjection $A \rightarrow R \cong A / I$. For an ideal $J \subset R$ we denote by $\tilde{J} \subset A$ the full preimage of $J$ in $A$. If $J$ is any ideal in a Noetherian ring of characteristic $p$, we use the standard notation $J^{\left[p^{e}\right]}$ to denote the ideal generated by the $p^{e}$-th powers of the elements (equivalently, a set of generators) of $J$. As always, $E$ denotes the injective hull of the residue field of the local ring $R$.

We first derive a characterization of those ideals in $R$ whose annihilators in $E$ are $\mathcal{F}(E)$-submodules of $E$.

5.2. Proposition. With notation as above in 5.1, let $J$ be an ideal of $R$ and let $M$ be the annihilator of $J$ in $E$, that is, $M=\{x \in E \mid J x=0\}$. Then $M$ is an $\mathcal{F}(E)$-submodule of $E$ if and only if $\left(I^{\left[p^{e}\right]}:_{A} I\right) \subset\left(\tilde{J}^{\left[p^{e}\right]}:_{A} \tilde{J}\right)$ for all $e$. 
Ideals whose annihilators in $H_{m}^{d}(R)$ are $\mathcal{F}\left(H_{m}^{d}(R)\right)$-submodules of $H_{m}^{d}(R)$ are called F-ideals in $\mathrm{S} 2$ and $\mathrm{S} 3$. For Gorenstein rings, $H_{m}^{d}(R)$ is isomorphic to $E$, so Proposition 5.2 above gives a characterization of F-ideals, without referring directly to $E$ or the Frobenius action on $E$. A different characterization of F-ideals, which does not require $R$ to be a homomorphic image of a regular ring, is proved in $\mathrm{S} 3$. $4.6]$.

Proof of Proposition 5.2. Whether or not an $R$-submodule $M$ of $E$ is an $\mathcal{F}(E)$ submodule of $E$ is independent of whether we regard $E$ as an $R$-module or as an $\hat{R}$-module. On the other hand, the module $M$ is the annihilator in $E$ of $J \hat{R}$; and because $\hat{A}$ is flat over $A$, the colon commutes with completion. Thus we can replace $R$ and $A$ by their respective completions, assuming without loss of generality that both $R$ and $A$ are complete.

Because $A$ surjects onto $R$, Proposition 3.4 ensures that the Frobenius structures on $E_{R}$ are the same considered over $R$ or over $A$. Thus the $R$-module $M$ is an $\mathcal{F}\left(E_{R}\right)$-submodule of $E_{R}$ if and only if it is stable under every $\phi_{e} \in \mathcal{F}^{e}\left(E_{R}\right)$ for all $e$, where we can take $\mathcal{F}^{e}\left(E_{R}\right)$ to mean $\operatorname{Hom}_{A}\left(A^{(e)} \otimes_{A} E_{R}, E_{R}\right)$. Thus we see that $M$ is an $\mathcal{F}\left(E_{R}\right)$-submodule of $E_{R}$ if and only if each $\phi_{e} \in \operatorname{Hom}_{A}\left(A^{(e)} \otimes_{A} E_{R}, E_{R}\right)$ induces a commutative diagram

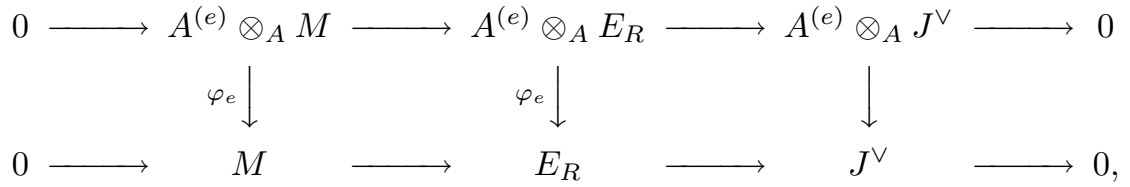

where for any $A$-module $M$, the notation $M^{\vee}$ denotes the Matlis dual $\operatorname{Hom}_{A}\left(M, E_{A}\right)$ (which, if $M$ is an $R$-module, can be identified with $\operatorname{Hom}_{R}\left(M, E_{R}\right)$ by the adjointness of tensor and hom coupled with the fact that $E_{R} \cong \operatorname{Hom}_{A}\left(R, E_{A}\right)$ ). Applying Matlis duality (over $A$ ) to this diagram and using the fact that the Frobenius functor and the Matlis duality functor commute for Artinian modules over $A[\mathrm{~L}] 4$.1], we see that $M$ is an $\mathcal{F}(E)$-submodule of $E$ if and only if every $\psi \in \operatorname{Hom}_{A}\left(R, A^{(e)} \otimes_{A} R\right)$ induces a commutative diagram

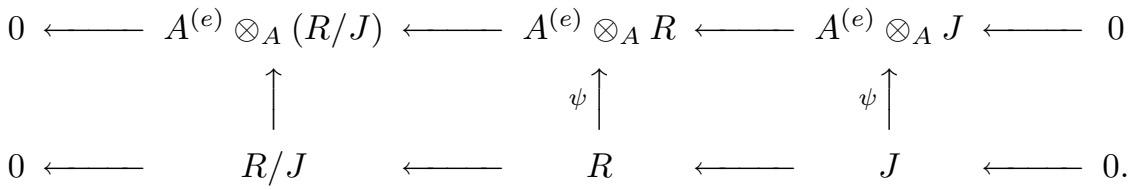

Thus $M$ is an $\mathcal{F}(E)$-submodule of $E$ if and only if every $\psi \in \operatorname{Hom}_{A}\left(R, A^{(e)} \otimes_{A} R\right)$ sends $J \subset R$ to $A^{(e)} \otimes_{A} J \subset A^{(e)} \otimes_{A} R$.

Recalling that $R \cong A / I$, we see that $A^{(e)} \otimes_{A} R=A / I^{\left[p^{e}\right]}$, so that

$$
\operatorname{Hom}_{A}\left(R, A^{(e)} \otimes_{A} R\right) \cong \operatorname{Hom}_{A}\left(A / I, A / I^{\left[p^{e}\right]}\right) \cong\left(I^{\left[p^{e}\right]}:_{A} I\right) / I^{\left[p^{e}\right]} .
$$

On the other hand, $A^{(e)} \otimes_{A} J=\tilde{J}^{\left[p^{e}\right]} / I^{\left[p^{e}\right]}$. So an element $\psi \in \operatorname{Hom}_{A}\left(R, A^{(e)} \otimes_{A} R\right)$ sends $J$ into $A^{(e)} \otimes_{A} J$ if and only if the element $\psi(1) \in\left(I^{\left[p^{e}\right]}:_{A} I\right) / I^{\left[p^{e}\right]}$ multiplies $\tilde{J}$ into $\tilde{J}^{\left[p^{e}\right]} / I^{\left[p^{e}\right]}$. Requiring this for every $\psi$, we see that $M$ is an $\mathcal{F}(E)$-submodule of $E$ if and only if $\tilde{J}\left(\left(I^{\left[p^{e}\right]}: I\right) / I^{\left[p^{e}\right]}\right)$ is contained in $\tilde{J}^{\left[p^{e}\right]} / I^{\left[p^{e}\right]}$, that is, if and only if $\left(I^{\left[p^{e}\right]}: I\right) \subset\left(\tilde{J}^{\left[p^{e}\right]}: \tilde{J}\right)$.

Next we show that the set of ideals $J \subset R$ whose annihilators in $E$ are $\mathcal{F}(E)$ submodules of $E$ is well behaved under localization. This is analogous to $\mathrm{S} 2$. 
4.8], where it is shown that F-ideals are well-behaved under localization in CohenMacaulay rings.

5.3. Proposition. With notation as in 5.1, for any prime ideal $P$ in $R$, let $E_{R_{P}}$ denote an injective hull of the residue field of the local ring $R_{P}$.

(1) Let $J \subset R$ be an ideal whose annihilator in $E$ is an $\mathcal{F}(E)$-submodule of $E$. Then the annihilator of $J_{P}$ in $E_{R_{P}}$ is an $\mathcal{F}\left(E_{R_{P}}\right)$-submodule of $E_{R_{P}}$.

(2) Let $J_{P} \subset R_{P}$ be an ideal such that its annihilator in $E_{R_{P}}$ is an $\mathcal{F}\left(E_{R_{P}}\right)$ submodule of $E_{R_{P}}$. Let $J \subset R$ be the full preimage of $J_{P}$ under the localization map $R \rightarrow R_{P}$. Then the annihilator of $J$ in $E$ is an $\mathcal{F}(E)$-submodule of $E$.

Proof. (1) By Proposition 5.2 we need to show that $\left(I_{P}^{\left[p^{e}\right]}: I_{P}\right) \subset\left(\tilde{J}_{P}^{\left[p^{e}\right]}: \tilde{J}_{P}\right)$; but this is immediate from $\left(I^{\left[p^{e}\right]}: I\right) \subset\left(\tilde{J}^{\left[p^{e}\right]}: \tilde{J}\right)$, since the colon and the inclusion are preserved by localization.

(2) By Proposition 5.2, we need to show that $\left(I^{\left[p^{e}\right]}:_{A} I\right) \subset\left(\tilde{J}^{\left[p^{e}\right]}: \tilde{J}\right)$. The condition that $\left(I^{\left[p^{e}\right]}: I\right) \subset\left(\tilde{J}^{\left[p^{e}\right]}: \tilde{J}\right)$ is equivalent to the vanishing of the $A$ module

$$
M=\frac{\left(I^{\left[p^{e}\right]}: I\right) \tilde{J}+\tilde{J}^{\left[p^{e}\right]}}{\tilde{J}\left[p^{e}\right]} .
$$

Because the annihilator of $J_{P}$ in $E_{R_{P}}$ is an $\mathcal{F}\left(E_{R_{P}}\right)$-module, we know that

$$
M_{\tilde{P}}=\frac{\left(I_{\tilde{P}}^{\left[p^{e}\right]}: I_{\tilde{P}}\right) \tilde{J}_{\tilde{P}}+\tilde{J}_{\tilde{P}}^{\left[p^{e}\right]}}{\tilde{J}_{\tilde{P}}^{\left[p^{e}\right]}}
$$

is zero, where $\tilde{P}$ is the preimage of $P$ in $A$.

Because $A$ is regular, the associated primes of $A / \tilde{J}^{\left[p^{e}\right]}$ are the same as the associated primes of $A / \tilde{J}$. Since $M \subset A / \tilde{J}^{\left[p^{e}\right]}$, every associated prime of $M$ is an associated prime of $\tilde{J}$. Furthermore, because $\tilde{J}$ is the full preimage of $\tilde{J}_{\tilde{P}}$ under $A \rightarrow A_{\tilde{P}}$, all associated primes of $\tilde{J}$, and hence all associated primes of $M$, are contained in $\tilde{P}$. In particular, $\tilde{P}$ is in the support of $M$, contradicting the vanishing of $M_{\tilde{P}}$.

\section{Maximal Frobenius submodules of $E$ And their anNihilators}

We have seen that annihilators of $\mathcal{F}(E)$-submodules of $E$ are well-behaved under localization, at least for rings that are homomorphic images of regular local rings. In this section we show that the annihilator of the tight closure of the zero module in $E$ also passes to localizations. For this, we will need to generalize our characterization of $0_{E}^{*}$ in Theorem 4.6 to include rings that may not be complete local domains.

6.1. Notation. As in Section 5, we assume throughout this section that $R \cong A / I$ is a homomorphic image of a regular local ring $A$ containing a field of characteristic $p$. Furthermore, we assume that $A$ (and hence $R$ ) is excellent. As always, $E$ (or $E_{R}$ ) denotes an injective hull of the residue field of $R$.

This section is devoted to proving the following two theorems.

6.2. Theorem. With notation as above in 6.1, assume furthermore that $R$ is reduced. Then $E_{R}$ admits a unique maximal $\mathcal{F}(E)$-submodule with respect to the 
property that its annihilator has positive height, and this submodule is the tight closure of zero in E. Furthermore, the tight closure of zero in $E$ is independent of whether we compute it over $R$ or over $\hat{R}$, and its annihilator in $\hat{R}$ is the expansion of its annihilator in $R$.

6.3. Theorem. With notation as above in 6.1, assume furthermore that $R$ is reduced, and let $P$ be a prime ideal of $R$. Let $J$ be the annihilator of $0_{E}^{*}$. Then the annihilator in $E_{R_{P}}$ of its localization $J R_{P}$ is $0_{E_{R_{P}}}^{*}$, where $E_{R_{P}}$ is an injective hull of the residue field of the local ring $R_{P}$.

6.4. Remark. An immediate consequence of Theorem 6.2 is a generalization of Theorem 4.1 characterizing strongly F-regular rings to the non-F-finite case. As before, the proof reduces immediately to the local case. Now if $R$ is strongly F-regular, it is a domain and, by Proposition $2.9,0_{E}^{*}=0$. This means that the maximal proper $\mathcal{F}(E)$-submodule of $E$ is trivial, so that $E$ is simple over $\mathcal{F}(E)$. Conversely, if $E$ is simple, then all proper $\mathcal{F}(E)$-submodules of $E$, including $0_{E}^{*}$, are zero. Again by $2.9, R$ is strongly F-regular.

Before proving these theorems, we need to develop a bit more theory. First, we need to know that the class of ideals in $\hat{R}$ that are annihilators in $E$ of $\mathcal{F}(E)$ submodules of $E$ is preserved under contraction to $R$.

6.5. Proposition. With notation as in 6.1 , let $J$ be an ideal of $\hat{R}$. If the annihilator of $J$ in $E$ is an $\mathcal{F}(E)$-submodule of $E$, then the annihilator in $E$ of its contraction $J \cap R$ is also an $\mathcal{F}(E)$-submodule of $E$.

The proof requires the following lemma.

6.6. Lemma. If $\tilde{J}$ is an ideal of $\hat{A}$, then $\tilde{J}^{\left[p^{e}\right]} \cap A=(\tilde{J} \cap A)^{\left[p^{e}\right]}$.

6.7. Remark. If $A$ is excellent, a result of Radu [R, Cor. 5] says that the natural ring homomorphism $\psi: A^{(e)} \otimes_{A} \hat{A} \stackrel{a \otimes b \mapsto a b^{p^{e}}}{\longrightarrow} \hat{A}$ is flat. Although Radu stated and proved this result for $e=1$, the same proof works for any $e$. The main ingredient of Radu's proof is the celebrated result of Popescu P1, P2. For an exposition of Popescu's result see $[\mathrm{Sw}$.

Proof of Lemma 6.6. Every element of $A^{(e)} \otimes_{A} \hat{A}$, raised to the $p^{e}$-th power, belongs to the subring $1 \otimes_{A} \hat{A} \cong \hat{A}$, hence the spectrums of $A^{(e)} \otimes_{A} \hat{A}$ and $\hat{A}$ are homeomorphic, i.e. $A^{(e)} \otimes_{A} \hat{A}$ is a local ring and $\psi$ is a local homomorphism. Every flat local homomorphism is faithfully flat and every faithfully flat homomorphism is injective; hence $\psi$ is injective. The image of $\psi$ is $A\left[\hat{A}^{p^{e}}\right]$, the $A$-subalgebra of $\hat{A}$ generated by the $p^{e}$-th powers of all the elements of $\hat{A}$. Thus $A^{(e)} \otimes_{A} \hat{A} \cong A\left[\hat{A}^{p^{e}}\right]$ and $\hat{A}$ is a flat $A\left[\hat{A}^{p^{e}}\right]$-module.

In $A^{(e)} \otimes_{A} \hat{A}$ we have that $\left(A^{(e)} \otimes_{A} \hat{J}\right) \cap\left(A^{(e)} \otimes_{A} A\right)=A^{(e)} \otimes_{A}(\tilde{J} \cap A)$ since for regular rings the Frobenius commutes with finite intersections [L, 1.0b]. As subsets of $A[\hat{A}]^{p^{e}}$, the set $\left(A^{(e)} \otimes_{A} \tilde{J}\right)$ is the ideal of $A[\hat{A}]^{p^{e}}$ generated by the $p^{e}$-th powers of all the elements of $\tilde{J}$, while $A^{(e)} \otimes_{A} A$ and $A^{(e)} \otimes_{A}(\tilde{J} \cap A)$ are just $A$ and $(\tilde{J} \cap A)^{\left[p^{e}\right]}$ respectively. We denote $\left(A^{(e)} \otimes_{A} \tilde{J}\right)$ by $\tilde{J}^{\left[p^{e}\right]^{\prime}}$. Thus $\tilde{J}^{\left[p^{e}\right]^{\prime}} \cap A=(\tilde{J} \cap A)^{\left[p^{e}\right]}$. But $\tilde{J}\left[p^{e}\right]^{\prime} \hat{A}=\tilde{J}\left[p^{e}\right]$. Since $\hat{A}$ is faithfully flat over $A\left[\hat{A}^{p^{e}}\right]$, we have that $I \hat{A} \cap A\left[\hat{A}^{p^{e}}\right]=I$ for every ideal $I$ of $A\left[\hat{A}^{p^{e}}\right]$; in particular, $\tilde{J}\left[p^{e}\right]^{\prime} \hat{A} \cap A\left[\hat{A}^{p^{e}}\right]=\tilde{J}\left[p^{e}\right] \cap A\left[\hat{A}^{p^{e}}\right]=\tilde{J}\left[p^{e}\right]^{\prime}$. Since $A \subset A\left[\hat{A}^{p^{e}}\right]$, we conclude that $\tilde{J}^{\left[p^{e}\right]} \cap A=\left(\tilde{J}^{\left[p^{e}\right]} \cap A\left[\hat{A}^{p^{e}}\right]\right) \cap A=\tilde{J}^{\left[p^{e}\right]^{\prime}} \cap A=$ $(\tilde{J} \cap A)^{\left[p^{e}\right]}$. 
Proof of Proposition 6.5. We denote by $\tilde{J} \subset \hat{A}$ the preimage of $J$ under the induced surjection $\hat{A} \rightarrow \hat{R}$. According to Proposition 5.2, we know that $\left(I^{\left[p^{e}\right]} \hat{A}: \hat{A}_{\hat{A}} I \hat{A}\right) \subset$ $\left(\tilde{J}^{\left[p^{e}\right]}:_{\hat{A}} \tilde{J}\right)$, and we need to show that $\left(I^{\left[p^{e}\right]}:_{A} I\right) \subset\left((\widetilde{J \cap R})^{\left[p^{e}\right]}:_{A}(\widetilde{J \cap R})\right)$. Because

$$
\widetilde{J \cap R}\left(I^{\left[p^{e}\right]}:_{A} I\right) \subset \tilde{J}\left(I^{\left[p^{e}\right]} \hat{A}:_{\hat{A}} I \hat{A}\right) \cap A \subset \tilde{J}^{\left[p^{e}\right]} \cap A,
$$

it is enough to show that $\tilde{J}^{\left[p^{e}\right]} \cap A=(\widetilde{J \cap R})^{\left[p^{e}\right]}$. But $\widetilde{J \cap R}=\tilde{J} \cap A$, so we are done by Lemma 6.6.

Using Proposition 6.5, we will show in Theorem 6.10 that annihilators in $\hat{R}$ of $\mathcal{F}(E)$-modules that are maximal with respect to certain properties are expanded from $R$. First we must prove Proposition 6.9 below, for which we need the following easy lemma.

6.8. Lemma. Let $S$ be a subset of the set of the minimal primes of $R$. Let $J$ be the intersection of the primary components of the zero ideal corresponding to the primes in this set $S$. Then the annihilator of $J$ in $E$ is an $\mathcal{F}(E)$-submodule of $E$.

Proof of Lemma 6.8. Let $a \in\left(I^{\left[p^{e}\right]}: I\right)$ and let $a^{\prime} \in \tilde{J}$. We need to show that $a a^{\prime} \in \tilde{J}^{\left[p^{e}\right]}$. We fix a primary decomposition of $I$ and let $\bar{J}$ be the intersection of all the primary ideals of this decomposition except those whose radicals are associated primes of $\tilde{J}$. Then $I=\tilde{J} \cap \bar{J}$ and there exists $a^{\prime \prime} \in \bar{J}$ which does not belong to any associated prime of $\tilde{J}$. We have that $a^{\prime \prime} a^{\prime} \in I$, hence $a^{\prime \prime} a^{\prime} a \in I^{\left[p^{e}\right]} \subset \tilde{J}^{\left[p^{e}\right]}$. Because $A$ is regular, the associated primes of $\tilde{J}^{\left[p^{e}\right]}$ are exactly those of $\tilde{J}$. Since $a^{\prime \prime}$ does not belong to any of them, $a^{\prime} a \in \tilde{J}^{\left[p^{e}\right]}$.

6.9. Proposition. Let $R$ be complete and reduced. Among the set of $\mathcal{F}(E)$-modules whose annihilators have positive height, there is a unique maximal element.

Proof of Proposition 6.9. Let $P_{1}, \ldots, P_{t}$ be the minimal primes of $R$ and let $\mathcal{J}_{i}$ be the intersection of all of them except $P_{i}$. By Lemma 6.8, the annihilator of $\mathcal{J}_{i}$ in $E$ is a proper $\mathcal{F}(E)$-submodule. By Lemma 4.5, this $\mathcal{F}(E)$-module is contained in a maximal proper $\mathcal{F}(E)$-submodule. We denote this maximal module by $M_{i}$. Because $M_{i}$ is proper, its annihilator $J_{i}$ is non-zero. Furthermore, because $J_{i} \subset \mathcal{J}_{i}$ and $R$ is reduced, $J_{i}$ belongs to all minimal primes of $R$ except $P_{i}$.

The module $M=\bigcap_{i=1}^{t} M_{i}$ is maximal among those $\mathcal{F}(E)$-modules whose annihilators are not contained in any minimal prime. Indeed, its annihilator is $\sum J_{i}$, which is easily seen to be not contained in any minimal prime. On the other hand, suppose that $M^{\prime}$ is a strictly larger $\mathcal{F}(E)$-module with the same property. Then $M^{\prime}$ is not contained in $M_{i}$ for some $i$ and the $\mathcal{F}(E)$-module $M^{\prime}+M_{i}$ has annihilator $\operatorname{Ann}_{R}\left(M^{\prime}\right) \cap \operatorname{Ann}_{R}\left(M_{i}\right)$, which is clearly not contained in the minimal prime $P_{i}$. Thus $M_{i}+M^{\prime}$ is a proper $\mathcal{F}(E)$-submodule of $E$ which is strictly larger than $M_{i}$, contrary to the maximality assumption on $M_{i}$.

Existence having been proven, uniqueness follows immediately: if $M^{\prime}$ and $M^{\prime \prime}$ are both maximal with respect to the property that their annihilators are not contained in any minimal prime, then consider the module $M^{\prime}+M^{\prime \prime}$. Its annihilator is $\operatorname{Ann}_{R}\left(M^{\prime}\right) \cap \operatorname{Ann}_{R}\left(M^{\prime \prime}\right)$, so is also not contained in any minimal prime. But by maximality, it follows that $M^{\prime}+M^{\prime \prime}=M^{\prime}=M^{\prime \prime}$.

We now show that annihilators in $\hat{R}$ of $\mathcal{F}(E)$-submodules of $E$ that are maximal in the sense of Proposition 6.9 are expanded from $R$. 
6.10. Theorem. With notation as in 6.1, assume furthermore that $R$ is reduced. Let $J$ be the annihilator in $\hat{R}$ of the unique $\mathcal{F}(E)$-submodule of $E$ maximal with respect to the property that its annihilator in $\hat{R}$ has positive height. Then $J$ is expanded from $R$; that is, $J=(J \cap R) \hat{R}$.

Proof. Let $\mathcal{M}$ be the maximal $\mathcal{F}(E)$-module whose annihilator $J$ in $\hat{R}$ is not contained in any minimal prime (of $\hat{R}$ ). By Proposition 6.5, the annihilator of $J \cap R$ in $E$ is an $\mathcal{F}(E)$-submodule of $E$; call this submodule $N$, and note that its annihilator in $\hat{R}$ is $(J \cap R) \hat{R}$. If $(J \cap R) \hat{R} \subsetneq J$, then $M \subsetneq N$. In other words, the annihilator of $N$ must be contained in some minimal prime of $\hat{R}$.

Because $R \rightarrow \hat{R}$ is faithfully flat, $\mathcal{I} \hat{R} \cap R=\mathcal{I}$ for every ideal $\mathcal{I}$ of $R$. So if $(J \cap R) \hat{R}$ is contained in a minimal prime of $\hat{R}$, then also $J \cap R$ is contained in a minimal prime of $R$. Because a primary decomposition of any ideal $J$ in $\hat{R}$ contracts to a primary decomposition of $J \cap R$, there must be a prime ideal $Q$ of $\hat{R}$ which is minimal over $J$ and which contracts to a minimal prime $P$ of $R$. Since $J$ is not contained in any minimal prime of $\hat{R}, Q$ is not a minimal prime of $\hat{R}$.

Localizing $\hat{R}$ at $Q$, we see that $J \hat{R}_{Q}$ is a proper non-zero ideal of $\hat{R}_{Q}$. By Proposition 5.3, its annihilator in $E_{\hat{R}_{Q}}$ is a proper non-zero $\mathcal{F}(E)$-module. In particular, $E_{\hat{R}_{Q}}$ is not a simple $\mathcal{F}\left(E_{\hat{R}_{Q}}\right)$-module.

Because $P$ is a minimal prime in a reduced ring, $P R_{P}$ is the zero ideal of $R_{P}$. Thus $(\hat{R})_{Q} \cong\left(\frac{R_{P}}{P R_{P}} \otimes_{R} \hat{R}\right)_{Q}$, so that $(\hat{R})_{Q}$ is a localization of a formal fiber ring of $R$. Since $R$ is excellent, this formal fiber ring is regular. But the injective hull of the residue field of a regular local ring $\mathcal{R}$ is a simple $\mathcal{F}\left(E_{\mathcal{R}}\right)$-module. This follows immediately from [S4, Theorem 2.6] after observing that a regular local ring $\mathcal{R}$ is Gorenstein, so that $E_{\mathcal{R}}$ can be identified with the top local cohomology module $H_{m}^{d}(\mathcal{R})$, and that $\mathcal{F}\left(E_{\mathcal{R}}\right) \cong \hat{\mathcal{R}}\{F\}$ as shown in Example 3.7. This contradicts the above observation that $E_{\hat{R}_{Q}}$ is not a simple $\mathcal{F}\left(E_{\hat{R}_{Q}}\right)$-module, finishing the proof.

We can now prove Theorem 6.2 on the existence of a unique maximal proper $\mathcal{F}(E)$-submodule of $E$ with respect to the property that its annihilator has positive height, and its characterization as the tight closure of the zero module in $E$.

Proof of Theorem 6.2. As $R$ is excellent, $\hat{R}$ is also reduced. So there is a unique proper $\mathcal{F}(E)$-submodule of $E$, call it $\mathcal{M}$, maximal with respect to the property that its annihilator is not contained in any minimal prime of $\hat{R}$. As pointed out in Remark 4.7 immediately following the proof of Theorem 4.6, this module $\mathcal{M}$ must be the tight closure of the zero module in $E$, computed over $\hat{R}$. Thus we have

$$
\left(0_{E}^{*}\right)_{R} \subset\left(0_{E}^{*}\right)_{\hat{R}}=\mathcal{M},
$$

where the subscripts $\hat{R}$ and $R$ on the tight closure modules indicate the ring. We need to verify two points: that this tight closure module is independent of whether we compute over $R$ or over $\hat{R}$ (i.e., that $\left(0_{E}^{*}\right)_{R}=\left(0_{E}^{*}\right)_{\hat{R}}$ ), and also that it is maximal with respect to its annihilator in $R$ not being contained in any minimal prime of $R$ (which is a priori not equivalent to the same assumption over $\hat{R}$ ).

Consider the $\mathcal{F}(E)$-module $\mathcal{M}$. We know from Theorem 6.10, that its annihilator in $\hat{R}$ is expanded from $R$. It follows that if $J$ denotes its annihilator in $R$, then its expansion $J \hat{R}$ is its annihilator in $\hat{R}$. Since $J$ and $J \hat{R}$ have the same height, the ideal $J$ cannot be contained in any minimal prime of $R$. Thus for any $\eta \in \mathcal{M}$, there is a $c \in R$ not in any minimal prime that annihilates the $\mathcal{F}(E)$-module generated 
by $\eta$. Now the argument described in the last paragraph of the proof of Theorem 4.4 applies here to show that $\mathcal{M} \subset\left(0_{E}^{*}\right)_{R}$. We conclude that $0_{E}^{*}$ is independent of whether the ring is considered to be $R$ or $\hat{R}$, verifying the first point outlined above.

It remains to show that $\mathcal{M}$ is maximal with respect to the property that its annihilator in $R$ is not contained in any minimal prime of $R$. But consider any $\mathcal{F}(E)$-module whose annihilator in $R$ is not contained in any minimal prime of $R$. Again, the same proof shows that $\mathcal{M}^{\prime} \subset 0_{E}^{*}$, so the proof that $0_{E}^{*}$ is the unique maximal proper such $\mathcal{F}(E)$-submodule is complete.

We can now prove Theorem 6.3, which guarantees that the annihilator of the tight closure of zero in $E$ behaves well under localization.

Proof of Theorem 6.3. Let $J$ be the annihilator, in $R$, of $0_{E}^{*}$. By Proposition 5.3, the annihilator of $J R_{P}$ in $E_{R_{P}}$ is an $\mathcal{F}\left(E_{R_{P}}\right)$-submodule of $E_{R_{P}}$, call it $N$. Clearly, the annihilator in $R_{P}$ of $N$ (which is $J R_{P}$ ) is not contained in any minimal prime. If $N$ is not maximal with respect to this property, suppose that $N$ is properly contained in some larger module $\mathcal{N}$, whose annihilator we will denote by $\mathcal{J} R_{P}$. Since $\mathcal{N} \subset \operatorname{Ann}_{E_{R_{P}}}\left(\operatorname{Ann}_{R_{P}}(\mathcal{N})\right)=\operatorname{Ann}_{E_{R_{P}}}\left(\mathcal{J} R_{P}\right)$, the proper inclusion $N \subset \mathcal{N}$ gives rise to a proper inclusion $\operatorname{Ann}_{E_{R_{P}}}\left(J R_{P}\right) \subset \operatorname{Ann}_{E_{R_{P}}}\left(\mathcal{J} R_{P}\right)$ and hence a proper inclusion $\mathcal{J} R_{P} \subset J R_{P}$.

Let $\mathcal{J}$ be the full preimage of $\mathcal{J} R_{P}$ in $R$. By Proposition 5.3, the annihilator of $\mathcal{J}$ in $E$ is an $\mathcal{F}(E)$-submodule of $E$. But because $\mathcal{J}$ is not contained in any minimal prime of $R$, this $\mathcal{F}(E)$ submodule must be contained in the unique maximal such $\mathcal{F}(E)$-module, whose annihilator is $J$. Thus $\mathcal{J}$ must contain $J$, so that $\mathcal{J} R_{P} \supset$ $J R_{P}$. This contradiction establishes Theorem 6.3.

\section{Consequences for the test ideal}

We now construct the ideal $\tilde{\tau}$ promised in the introduction. For any ring $R$ of prime characteristic $p$, define $\tilde{\tau}$ to be the annihilator, in $R$, of the tight closure of the zero module in $E$, where, as always, $E$ is the direct sum, over all maximal ideals $m$ in Spec $R$, of the injective hulls $E(R / m)$ of $R / m$. In symbols,

$$
\tilde{\tau}(R)=\operatorname{Ann}_{R}\left(0_{E}^{*}\right),
$$

where

$$
E=\bigoplus_{m \in \max \operatorname{Spec} R} E(R / m) .
$$

We often write $\tilde{\tau}$ when $R$ is clear from context.

It is easy to see that the tight closure of zero in $E$ is the direct sum of the tight closures of zero in each $E(R / m)$ (see the proof of 4.1). Thus its annihilator $\tilde{\tau}$ is the intersection

$$
\bigcap \operatorname{Ann}_{R}\left(0_{E(R / m)}^{*}\right) \text {. }
$$

We first summarize what we have essentially already proved in Section 6 .

7.1. Theorem. Let $R$ be a reduced ring that is a homomorphic image of an excellent regular local ring of prime characteristic $p$. Then:

(1) $\tilde{\tau}$ has positive height.

(2) For every prime ideal $P$ of $R, \tilde{\tau} R_{P}=\tilde{\tau}\left(R_{P}\right)$. 
(3) $\tilde{\tau} \hat{R}=\tilde{\tau}(\hat{R})$.

(4) $\tilde{\tau}$ is contained in the completely stable test ideal of $R$.

(5) $\tilde{\tau}$ defines the non-strongly F-regular locus of $R$ (where strong F-regularity is defined as in Remark 2.8 even if $R$ is not F-finite).

(6) If $0_{E}^{*}=0_{E}^{* f g}$, then $\tilde{\tau}$ is the test ideal, i.e. $\tilde{\tau}(R)=\tau(R)$; in particular, the test ideal would be the same as the completely stable test ideal, and by (3) the test ideal would commute with completion.

(7) If, for every prime ideal $P$ of $R, 0_{E_{R_{P}}}^{*}=0_{E_{R_{P}}}^{* f g}$ (computed over $R_{P}$ ), then the test ideal of $R$ commutes with localization: for every multiplicative set $\mathcal{U} \subset R$, $\tau\left(R\left[\mathcal{U}^{-1}\right]\right)=\tau(R) R\left[\mathcal{U}^{-1}\right]$; in particular, the weakly F-regular locus would be open and its complement would be defined by the test ideal.

Proof of Theorem 7.1. Here, $\tilde{\tau}=\operatorname{Ann}_{R}\left(0_{E}^{*}\right)$, where $E$ is an injective hull of the residue field of $R$. By Theorem 6.2, $\tilde{\tau}$ has positive height, proving (1). By Theorem $6.3, \tilde{\tau}\left(R_{P}\right)=\tilde{\tau} R_{P}$, so that (2) holds, whereas (3) follows from the last sentence of Theorem 6.2. For (4), note that since $0_{E}^{* f g} \subset 0_{E}^{*}, \tilde{\tau} \subset \operatorname{Ann}_{R}\left(0_{E}^{* f g}\right)=\tau$, the test ideal of $R$. So for any prime ideal $P$ of $R$, we have

$$
\tilde{\tau} R_{P}=\tilde{\tau}\left(R_{P}\right) \subset \tau\left(R_{P}\right), \quad \text { and } \quad \tilde{\tau} \widehat{R_{P}}=\tilde{\tau}\left(\widehat{R_{P}}\right) \subset \tau\left(\widehat{R_{P}}\right),
$$

where $\widehat{R_{P}}$ denotes the completed local ring of $R$ at $P$. This means that the image of any element of $\tilde{\tau}$ in a local or a completed local ring of $R$ is in the test ideal of the appropriate ring. Thus $\tilde{\tau}$ is contained in the completely stable test ideal of $R$.

To prove (5), recall that $P$ is in the strongly F-regular locus if and only if $0_{E_{R_{P}}}^{*}=0$. By (2), this holds if and only if $\tilde{\tau}\left(R_{P}\right)=R_{P}$. Since $\tilde{\tau}\left(R_{P}\right)=\tilde{\tau} R_{P}$, a prime $P$ fails to be in the strongly F-regular locus if and only if it contains $\tilde{\tau}$.

Item (6) follows immediately from the characterization of the test ideal as the annihilator of $0_{E}^{* f g}$, whereas (7) follows from (2) together with the fact that an element $c$ is in the test ideal of $R$ if and only if its image in each localization at a maximal ideal $m$ of $R$ is in the test ideal of $R_{m}$ [HH1, 6.1a].

We expect that the ideal $\tilde{\tau}$ has the properties listed in Theorem 7.1 for an arbitrary reduced excellent ring. So far, however, we have been unable to prove this in complete generality. However, we do have the following theorem.

7.2. Theorem. Let $R$ be a reduced ring essentially of finite type over an excellent regular local ring of prime characteristic $p$. Then:

(1) $\tilde{\tau}$ has positive height.

(2) For every prime $P \subset R, \tilde{\tau} R_{P} \subset R_{P}$ is the annihilator in $R_{P}$ of $0_{E_{R_{P}}}^{*}$.

(3) For every multiplicative system $\mathcal{U} \subset R, \tilde{\tau} R\left[\mathcal{U}^{-1}\right]=\tilde{\tau}\left(R\left[\mathcal{U}^{-1}\right]\right)$.

(4) For every prime ideal $P$ of $R, \tilde{\tau} \widehat{R_{P}}=\tilde{\tau}\left(\widehat{R_{P}}\right)$.

(5) $\tilde{\tau}$ is contained in the completely stable test ideal of $R$.

(6) $\tilde{\tau}$ defines the non-strongly F-regular locus of $R$ (where strong F-regularity is defined as in Remark 2.8 even if $R$ is not F-finite).

(7) If $0_{E_{R_{P}}}^{*}=0_{E_{R_{P}}}^{* f g}$ (computed over $R_{P}$ ) for every maximal ideal $P \subset R$, then $\tilde{\tau}$ is the test ideal of $R$, i.e. $\tilde{\tau}(R)=\tau(R)$; in particular, the test ideal would be the same as the completely stable test ideal, and it would commute with completion at maximal ideals.

(8) If $0_{E_{R_{P}}}^{*}=0_{E_{R_{P}}}^{* f g}$ (computed over $R_{P}$ ) for every prime ideal $P \subset R$, the test ideal of $R$ commutes with localization: for every multiplicative set $\mathcal{U} \subset R$, 
$\tau\left(R\left[\mathcal{U}^{-1}\right]\right)=\tau(R) R\left[\mathcal{U}^{-1}\right]$; in particular, the weakly F-regular locus would be open and its complement would be defined by the test ideal.

The proof of Theorem 7.2 uses the idea of a homogenization of a finitely generated algebra over a ring $K$. Homogenizing a $K$-algebra is the algebraic counterpart to taking the projective closure of an affine scheme over $K$ in projective space over $K$. We now briefly recall this idea before embarking on the proof.

7.3. If $R=\frac{K\left[x_{1}, \ldots, x_{d}\right]}{I}$ is a finitely generated $K$-algebra, then the homogenization $\mathcal{R}=\frac{K\left[X_{0}, \ldots, X_{d}\right]}{\mathcal{I}}$ of $R$ is a finitely generated graded $K$-algebra which maps onto $R$ via the map setting $X_{0}$ to 1 and $X_{i}$ to $x_{i}$. Geometrically, if we think of $R$ as the affine coordinate ring of a closed subscheme of $\mathbb{A}_{K}^{n}$, then $\mathcal{R}$ is the homogeneous coordinate ring of its projective closure in $\mathbb{P}_{K}^{n}$. Algebraically, $R$ may be described as the zeroth graded piece of the $\mathbb{Z}$-graded ring $\mathcal{R}\left[\frac{1}{X_{0}}\right]$, in which case the variables $x_{i}$ correspond to the fractions $\frac{X_{i}}{X_{0}}$. (Of course, $\mathcal{R}$ depends on the choice of presentation of $R$, but we assume our coordinates fixed and refer freely to "the" homogenization of $R$ ).

If $J$ is any ideal of $R$, then its homogenenization $\mathcal{J}$ is the (saturated) homogeneous ideal of $\mathcal{R}$ which corresponds to $J$ under the map $\mathcal{R} \rightarrow R$. Explicitly, $\mathcal{J}$ is the homogeneous ideal of $\mathcal{R}$ generated by the homogenizations of all elements of $J$, where the homogenization of a polynomial $f\left(x_{1}, \ldots, x_{d}\right)=\sum_{i=0}^{r} g_{i}\left(x_{1}, \ldots, x_{d}\right)$ (with $g_{i}$ homogeneous of degree $i$ in $x_{1}, \ldots, x_{d}$ and $g_{r} \neq 0$ ) is the homogeneous polynomial $F_{i}\left(X_{0}, \ldots X_{d}\right)=\sum_{i=0}^{r} X_{0}^{r-i} g_{i}\left(X_{1}, \ldots, X_{d}\right)$. We call $J$ the dehomogenization of $\mathcal{J}$. Since the dimension of a subscheme of $\operatorname{Spec} R$ is unchanged when we take its closure in $\operatorname{Proj} \mathcal{R}$, the heights of $J$ and $\mathcal{J}$ are the same. Of course, $\mathcal{J}$ is prime (respectively, radical) if and only if $J$ is prime (radical). Furthermore, when $\mathcal{P}$ is the homogenization of a prime ideal $P$ in $R$, the local ring $R_{P}$ is the zeroth graded piece of the $\mathbb{Z}$-graded ring $\mathcal{R}_{\langle\mathcal{P}\rangle}$, where $\mathcal{R}_{\langle\mathcal{P}\rangle}$ denotes the localization of $\mathcal{R}$ at the multiplicative system of homogeneous elements of $\mathcal{R}$ not in $\mathcal{P}$. The natural map $R_{P} \rightarrow \mathcal{R}_{\langle\mathcal{P}\rangle}$ is faithfully flat, since each graded piece of $\mathcal{R}_{\langle\mathcal{P}\rangle}$ is free over the zeroth graded piece, and clearly $\mathcal{R}_{\langle\mathcal{P}\rangle} \rightarrow \mathcal{R}_{\mathcal{P}}$ is flat, being a localization map. For general information on the process of homogenization, see [Ha, pp. 76-78].

Proof of 7.2. We first note that if $R$ is any ring of prime characteristic, and if there exists an ideal $\tau^{\prime}$ of $R$ whose localization at each maximal ideal $m$ is the annihilator, in $R_{m}$, of the tight closure of the zero module in $E(R / m)$, then $\tau^{\prime}$ must coincide with $\tilde{\tau}$. Indeed, since $\tilde{\tau} \subset \operatorname{Ann}_{R}\left(0_{E(R / m)}^{*}\right)$ for each $m$, we see that $\tilde{\tau} R_{m} \subset \operatorname{Ann}_{R_{m}}\left(0_{E(R / m)}^{*}\right)=\tau^{\prime} R_{m}$ for all $m$, whence $\tilde{\tau} \subset \tau^{\prime}$. On the other hand, since $\tau^{\prime} R_{m}=\operatorname{Ann}_{R_{m}}\left(0_{E(R / m)}^{*}\right)$ and since the elements of $R \backslash m$ act injectively on $E(R / m)$, we see that $\tau^{\prime} \subset \operatorname{Ann}_{R}\left(0_{E(R / m)}^{*}\right)$ for all $m$, whence $\tau^{\prime} \subset$ $\bigcap_{m} \operatorname{Ann}_{R}\left(0_{E(R / m)}^{*}\right)=\tilde{\tau}$. Thus $\tau^{\prime}=\tilde{\tau}$.

We now define an ideal $\tau^{\prime}$ for a finitely generated algebra $R$ over $K$ and show that it has the desired properties. Let $\mathcal{R}$ be the homogenization of $R$, and let $E_{\mathcal{R}}$ be the injective hull of the $\mathcal{R}$-module $\mathcal{R} / \mathcal{M}$, where $\mathcal{M}$ denotes the unique maximal homogeneous ideal of $\mathcal{R}$. Let $\mathcal{T}$ be the annihilator, in $\mathcal{R}$, of the tight closure of the zero module in $E_{\mathcal{R}}$. It is easy to check that this tight closure module is graded, whence $\mathcal{T}$ is a homogeneous ideal. We set $\tau^{\prime}(R)$ to be the dehomogenization of $\mathcal{T}$, that is, $\tau^{\prime}(R)$ is the zeroth graded part of the $\mathbb{Z}$-graded module $\mathcal{T} \mathcal{R}\left[\frac{1}{X_{0}}\right]$. 
If $R$ is a localization of a finitely generated algebra $S$ over $K$, then we define $\tau^{\prime}(R)$ to be the expansion of $\tau^{\prime}(S)$ to the localization $R$.

We now need to show that $\tau^{\prime}(R)$ has the purported properties, whence it will follow that $\tau^{\prime}=\tilde{\tau}$. It is enough to check all these properties when $R$ is a finitely generated algebra over $K$; the case where $R$ is essentially of finite type follows easily because the prime ideals of an algebra essentially of finite type are a subset of those in an algebra of finite type. From the definition of $\tau^{\prime}(R)$, it is not even clear that it is well-defined, as it seems to depend on a choice of presentation for $R$. However, well-definedness will follow immediately once we have shown (2), since an ideal of $R$ is uniquely defined by its localizations at all maximal ideals.

(1) Since ideals of $R$ and their homogeneous counterparts in $\mathcal{R}$ have the same height, it is enough to check that $\mathcal{T}$ is not contained in any minimal prime of $\mathcal{R}$. If the homogeneous ideal $\mathcal{T}$ is contained in a minimal prime of $\mathcal{R}$, then its expansion $\mathcal{T} \mathcal{R}_{\mathcal{M}}$ to $\mathcal{R}_{\mathcal{M}}$ is contained in a minimal prime of $\mathcal{R}_{\mathcal{M}}$. But this is prohibited by Theorem 6.2. Indeed, $\mathcal{T} \mathcal{R}_{\mathcal{M}}$ is the annihilator in $\mathcal{R}_{\mathcal{M}}$ of $0_{E_{\mathcal{R}_{\mathcal{M}}}}^{*}$, that is, $\mathcal{T}=\tilde{\tau}\left(\mathcal{R}_{\mathcal{M}}\right)$ in the sense of Theorem 7.1. This is easy to see upon observing that since elements of $\mathcal{R}$ not in $\mathcal{M}$ act invertibly on $E_{\mathcal{R}}=E_{\mathcal{R}_{\mathcal{M}}}$, the tight closure of zero in $E_{\mathcal{R}}$ is independent of whether the computation is carried out over $\mathcal{R}$ or over $\mathcal{R}_{\mathcal{M}}$.

(2) We first show that for any prime ideal $P$ of $R$, the annihilator of $\tau^{\prime} R_{P}$ in $E_{R_{P}}$ is an $\mathcal{F}\left(E_{R_{P}}\right)$-module. Set $A$ to be the polynomial ring $K\left[x_{1}, \ldots, x_{d}\right]$ mapping onto $R$ with kernel $I$, and set $\mathcal{A}$ to be the polynomial ring $K\left[X_{0}, \ldots, X_{d}\right]$ mapping onto $\mathcal{R}$ with kernel $\mathcal{I}$, so that $\mathcal{I}$ is the homogenization of $I$. As before, let $\tilde{J}$ denote the full preimage in $A$ (or $\mathcal{A}$ ) of an ideal $J$ in $R$ (or $\mathcal{R}$ ) 2 Now, because $\mathcal{T}$ is the annihilator, in $\mathcal{R}$, of an $\mathcal{F}\left(E_{\mathcal{R}}\right)$-submodule of $E_{\mathcal{R}}$, we have

$$
\left(\mathcal{I}^{\left[p^{e}\right]}:_{\mathcal{A}} \mathcal{I}\right) \subset\left(\tilde{\mathcal{T}}^{\left[p^{e}\right]}:_{\mathcal{A}} \tilde{\mathcal{T}}\right)
$$

for all $e$ by Proposition 5.2. (Strictly speaking, Proposition 5.2 yields this inclusion after localizing at the unique homogeneous maximal ideal of $\mathcal{A}$, but as $\mathcal{I}$ and $\tilde{\mathcal{T}}$ are homogeneous, it follows without localizing as well.)

Inverting $X_{0}$ (which commutes with the colon) and looking at the zeroth graded piece of the corresponding inclusion of ideals (that is, dehomogenizing), we have the inclusion

$$
\left(I^{\left[p^{e}\right]}:_{A} I\right) \subset\left({\widetilde{\tau^{\prime}}}^{\left[p^{e}\right]}::_{A} \widetilde{\tau^{\prime}}\right)
$$

of ideals in $A$. Now localizing at any prime ideal $\tilde{P}$ of $A$, we have

$$
\left(\left(I A_{\tilde{P}}\right)^{\left[p^{e}\right]}:_{A_{\tilde{P}}} I A_{\tilde{P}}\right) \subset\left(\widetilde{\tau^{\prime} A_{\tilde{P}}}{ }^{\left[p^{e}\right]}::_{\tilde{P}} \widetilde{\tau^{\prime} A_{\tilde{P}}}\right) .
$$

By Proposition 5.2, it follows that $\tau^{\prime} R_{P}$ annihilates some $\mathcal{F}\left(E_{R_{P}}\right)$-module, where $P$ is the prime ideal of $R$ corresponding to $\tilde{P}$ in $A$. It remains only to show that this $\mathcal{F}\left(E_{R_{P}}\right)$-module, call it $\mathcal{N}$, is $0_{E_{R_{P}}}^{*}$.

Clearly $\tau^{\prime} R_{P}$ is not contained in any minimal prime of $R$. By Theorem 6.2, $0_{E_{R_{P}}}^{*}$ is the unique maximal module with respect to its annihilator not being contained in a minimal prime, so $\mathcal{N} \subset 0_{E_{R_{P}}}^{*}$. Because $\mathcal{N}=\operatorname{Ann}_{E_{P}}\left(\tau^{\prime} R_{P}\right)$ and because $0_{E_{P}}^{*}=\operatorname{Ann}_{E_{P}}\left(\operatorname{Ann}_{R_{P}}\left(0_{E_{R_{P}}}^{*}\right)\right.$ ) (the latter fact following from 6.10), this inclusion can be written $\operatorname{Ann}_{E_{P}}\left(\tau^{\prime} R_{P}\right) \subset \operatorname{Ann}_{E_{P}}\left(\operatorname{Ann}_{R_{P}}\left(0_{E_{R_{P}}}^{*}\right)\right)$. If this inclusion is strict,

\footnotetext{
${ }^{2}$ We apologize for the overlap of notation with $\tilde{\tau}$, although this is not likely to cause undue confusion.
} 
then of course also the inclusion $\operatorname{Ann}_{R_{P}}\left(0_{E_{R_{P}}}\right) \subset \tau^{\prime} R_{P}$ must be strict. Expanding to the faithfully flat overring $\mathcal{R}_{\langle\mathcal{P}\rangle}$, where $\mathcal{R}_{\langle\mathcal{P}\rangle}$ is the localization of $\mathcal{R}$ at the multiplicative system of homogeneous elements of $\mathcal{R}$ not in $\mathcal{P}$, we have a strict inclusion

$$
\left(\operatorname{Ann}_{R_{P}}\left(0_{E_{R_{P}}}^{*}\right)\right) \mathcal{R}_{\langle\mathcal{P}\rangle} \subsetneq \tau^{\prime} \mathcal{R}_{\langle\mathcal{P}\rangle}=\mathcal{T} \mathcal{R}_{\langle\mathcal{P}\rangle} .
$$

Since both ideals above are graded, the strict inclusion is preserved after further expansion to $\mathcal{R}_{\mathcal{P}}$ :

$$
\left(\operatorname{Ann}_{R_{P}}\left(0_{E_{R_{P}}}^{*}\right)\right) \mathcal{R}_{\mathcal{P}} \subsetneq \mathcal{T} \mathcal{R}_{\mathcal{P}} .
$$

Now, because $0_{E_{R_{P}}}^{*}$ is an $\mathcal{F}\left(E_{R_{P}}\right)$-module, we have, by Proposition 5.2,

$$
\left.\left(\left(I A_{\tilde{P}}\right)^{\left[p^{e}\right]}:_{A_{\tilde{P}}} I A_{\tilde{P}}\right) \subset\left(\left(\widetilde{\operatorname{Ann} \widetilde{R_{P}\left(0_{E_{R_{P}}^{*}}^{*}\right.}}\right)\right)^{\left[p^{e}\right]}:_{A_{\tilde{P}}}\left(\operatorname{Ann}_{R_{P}\left(0_{E_{R_{P}}^{*}}^{*}\right.}\right)\right),
$$

and because $A_{\tilde{P}} \rightarrow \mathcal{A}_{\tilde{\mathcal{P}}}$ is flat, we have the same after expanding to $\mathcal{A}_{\tilde{\mathcal{P}}}$ :

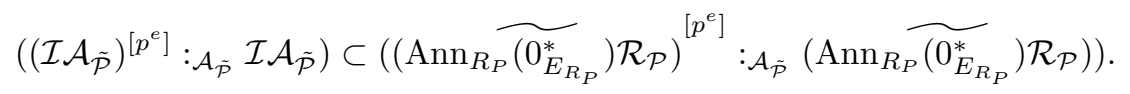

This says that $\operatorname{Ann}_{R_{P}}\left(0_{E_{R_{P}}}^{*}\right) \mathcal{R}_{\mathcal{P}}$ annihilates an $\mathcal{F}\left(E_{\mathcal{R}_{\mathcal{P}}}\right)$-submodule of $E_{\mathcal{R}_{\mathcal{P}}}$. But since $\operatorname{Ann}_{R_{P}}\left(0_{E_{R_{P}}^{*}}\right) \mathcal{R}_{\mathcal{P}}$ is not contained in any minimal prime, it must therefore contain $\mathcal{T}_{\mathcal{P}}$, which is the annihilator of the unique maximal $\mathcal{F}$-submodule whose annihilator has this property. This proves the inclusions above cannot be strict, so that $0_{E_{R_{P}}}^{*}=\mathcal{N}$. We conclude that the annihilator in $R_{P}$ of $0_{E_{R_{P}}}^{*}$ is $\tau^{\prime} R_{P}$, and the proof of (2) is complete. In particular, $\tau^{\prime}=\tilde{\tau}$.

The proof of (3) is easy now, because of the way in which $\tau^{\prime}$ is defined for an algebra $R$ essentially of finite type over $K$. Indeed, suppose that $R=S\left[\mathcal{V}^{-1}\right]$, where $\mathcal{V}$ is a multiplicative system in a finitely generated $K$-algebra $S$. Let $\mathcal{U}$ denote a multiplicative system in $R$, and by abuse of notation, also denote its preimage in $S$. Then

$$
\tilde{\tau}\left(R\left[\mathcal{U}^{-1}\right]\right):=\tilde{\tau}(S) S\left[\mathcal{V}^{-1}\right]\left[\mathcal{U}^{-1}\right]=\tilde{\tau}\left(S\left[\mathcal{V}^{-1}\right]\right) S\left[\mathcal{V}^{-1}\right]\left[\mathcal{U}^{-1}\right]=\tilde{\tau}(R) R\left[\mathcal{U}^{-1}\right],
$$

and the proof of $(3)$ is complete.

Now the properties (4)-(8) follow exactly as in the proof of Theorem 7.1.

\section{Some Cases Where Conjecture 2.6 holds}

The results of the preceding section focus our attention on the conjecture raised in the introduction.

8.1. Conjecture. If $N$ is a submodule of an Artinian module $M$ over an excellent local ring, then the tight closure of $N$ in $M$ is the union of the tight closures of $N \cap M^{\prime}$ in $M^{\prime}$, where $M^{\prime}$ ranges over all finitely generated submodules of $M$.

Even proving this when $M$ is the injective hull of the residue field of a complete local weakly F-regular ring and $N$ is its zero submodule would enable us to deduce that the weakly F-regular locus is open, at least for reasonable rings.

In this section, we establish this conjecture in two cases. In Theorem 8.8, we prove that if a Cohen-Macaulay local ring is Gorenstein on its punctured spectrum, then $0_{E}^{*}=0_{E}^{* f g}$, and in Theorem 8.12 we show that if a local ring has an isolated singularity, then $0_{M}^{*}=0_{M}^{* f g}$ for every Artinian module $M$. 
8.2. Notation. Throughout this section, $R$ denotes a local Noetherian ring with maximal ideal $m$. If $M$ is an Artinian $R$-module, $M_{-v} \subset M$ denotes the submodule annihilated by $m^{v}$.

8.3. Lemma. Let $M$ be an Artinian R-module. Assume that for every $v$ there exists $v^{\prime}$ such that for all e, the natural map

$$
\operatorname{Tor}_{1}^{R}\left(R^{(e)},\left(M / M_{-v}\right)_{-\left(v^{\prime}-v\right)}\right) \rightarrow \operatorname{Tor}_{1}^{R}\left(R^{(e)}, M / M_{-v}\right)
$$

is surjective. Then $0_{M}^{*}=0_{M}^{* f g}$. (Here, $R^{(e)}$ is considered as a right $R$-module.)

Proof. The commutative diagram

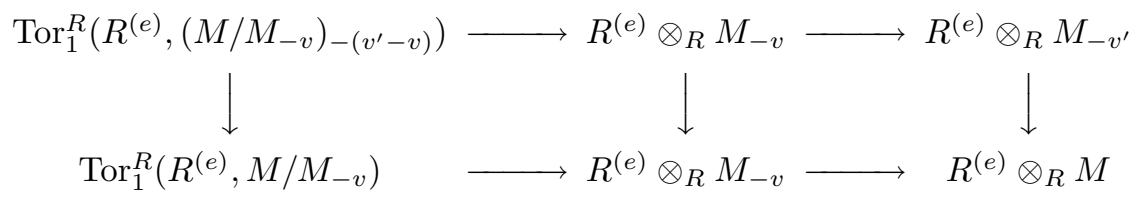

with exact rows shows that the natural map $R^{(e)} \otimes_{R} M_{-v^{\prime}} \rightarrow R^{(e)} \otimes_{R} M$ induces an isomorphism between the images of $R^{(e)} \otimes_{R} M_{-v}$ in both modules. Thus if $x \in 0_{M}^{*}$ is annihilated by $m^{v}$, then $x \in 0_{M_{-v^{\prime}}}^{*}$.

8.4. Proposition. For every Artinian $R$-module $M$ and integers $t, v, i \geq 0$ there exist integers $\psi(M, t, i)$ and $\phi(M, t, v, i)>v$ such that:

(a) For all $R$-modules $N$ annihilated by $m^{t}$ and all $v^{\prime}>\psi(M, t, i)$, the natural map $\operatorname{Tor}_{i}^{R}\left(N, M_{-v^{\prime}}\right) \rightarrow \operatorname{Tor}_{i}^{R}(N, M)$ is surjective.

(b) For all $R$-modules $N$ annihilated by $m^{t}$ and all $v^{\prime}>\phi(M, t, v, i)$, the map $\operatorname{Tor}_{i}^{R}\left(N, M_{-v^{\prime}}\right) \rightarrow \operatorname{Tor}_{i}^{R}(N, M)$ induced by the natural inclusion $M_{-v^{\prime}} \rightarrow M$ induces an isomorphism on the images of $\operatorname{Tor}_{i}^{R}\left(N, M_{-v}\right)$ in both modules.

Proof. We use induction on $t$. If $t=1$, then $N$ is a direct sum of copies of the residue field $k$, so we can replace $N$ by $k$. Since $\operatorname{Tor}_{i}^{R}(k, M)$ is Artinian and annihilated by $m$, it has finite length. Since it is the direct limit of $\operatorname{Tor}_{i}^{R}\left(N, M_{-v^{\prime}}\right)$ over all $v^{\prime}$, there is some $\psi(M, 1, i)$ such that for all $v^{\prime}>\psi(M, 1, i)$ the natural map $\operatorname{Tor}_{i}^{R}\left(N, M_{-v^{\prime}}\right) \rightarrow \operatorname{Tor}_{i}^{R}(N, M)$ is surjective. This proves (a) for $t=1$. Now it is enough to prove the following two claims:

(1) If for a fixed $t, \psi(M, t, i)$ exists for all $M$ and $i$, then $\phi(M, t, v, i)$ also exists for all $M, v$ and $i$, and

(2) If for a given $t, \phi(M, t, v, i)$ exists for all $M, v, i$, then $\psi(M, t+1, i)$ also exists for all $M, t, i$.

Proof of (1): The short exact sequences

$$
\begin{gathered}
0 \rightarrow M_{-v} \rightarrow M \rightarrow M / M_{-v} \rightarrow 0 \\
0 \rightarrow M_{-v} \rightarrow M_{-v^{\prime}} \rightarrow\left(M / M_{-v}\right)_{-\left(v^{\prime}-v\right)} \rightarrow 0,
\end{gathered}
$$

produce exact sequences

$$
\begin{gathered}
\operatorname{Tor}_{i+1}^{R}\left(N, M / M_{-v}\right) \rightarrow \operatorname{Tor}_{i}^{R}\left(N, M_{-v}\right) \rightarrow \operatorname{Tor}_{i}^{R}(N, M), \\
\operatorname{Tor}_{i+1}^{R}\left(N,\left(M / M_{-v}\right)_{-\left(v^{\prime}-v\right)}\right) \rightarrow \operatorname{Tor}_{i}^{R}\left(N, M_{-v}\right) \rightarrow \operatorname{Tor}_{i}^{R}\left(N, M_{-v^{\prime}}\right),
\end{gathered}
$$

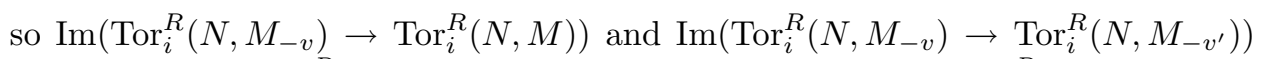
are quotients of $\operatorname{Tor}_{i}^{R}\left(N, M_{-v}\right)$ by the images of, respectively, $\operatorname{Tor}_{i+1}^{R}\left(N, M / M_{-v}\right)$ and $\operatorname{Tor}_{i+1}^{R}\left(N,\left(M / M_{-v}\right)_{-\left(v^{\prime}-v\right)}\right)$. Setting $\phi(M, t, v, i)=v+\psi\left(M / M_{-v}, t, i+1\right)$, 
we see that for $v^{\prime}>\psi(M, t, v, i)$, the module $\operatorname{Tor}_{i+1}^{R}\left(N,\left(M / M_{-v}\right)_{-\left(v^{\prime}-v\right)}\right)$ surjects onto $\operatorname{Tor}_{i+1}^{R}\left(N, M / M_{-v}\right)$, which implies the required isomorphism. This proves (1).

Proof of (2): Let $N$ be annihilated by $m^{t+1}$. Then there exists an exact sequence $0 \rightarrow N^{\prime} \rightarrow N \rightarrow N^{\prime \prime} \rightarrow 0$ with $N^{\prime}, N^{\prime \prime}$ annihilated by $m^{t}$. $\operatorname{Tor}_{i}^{R}\left(N^{\prime}, M_{-v}\right)$, $\operatorname{Tor}_{i}^{R}\left(N^{\prime \prime}, M_{-v}\right)$ and $\operatorname{Tor}_{i-1}^{R}\left(N^{\prime}, M_{-v}\right)$, for $v>\psi(M, t, i), \psi(M, t, i-1)$, surject onto, respectively, $\operatorname{Tor}_{i}^{R}\left(N^{\prime}, M\right), \operatorname{Tor}_{i}^{R}\left(N^{\prime \prime}, M\right)$ and $\operatorname{Tor}_{i-1}^{R}\left(N^{\prime}, M\right)$. And the modules $\operatorname{Im}\left(\operatorname{Tor}_{i}^{R}\left(N^{\prime}, M_{-v}\right)\right), \operatorname{Im}\left(\operatorname{Tor}_{i}^{R}\left(N^{\prime \prime}, M_{-v}\right)\right)$ and $\operatorname{Im}\left(\operatorname{Tor}_{i-1}^{R}\left(N^{\prime}, M_{-v}\right)\right)$, where $\operatorname{Im}$ means the image in $\operatorname{Tor}_{*}^{R}\left(-, M_{-v^{\prime}}\right)$, are respectively isomorphic to $\operatorname{Tor}_{i}^{R}\left(N^{\prime}, M\right)$, $\operatorname{Tor}_{i}^{R}\left(N^{\prime \prime}, M\right)$ and $\operatorname{Tor}_{i}^{R}\left(N^{\prime}, M\right)$, for $v^{\prime}>v, \phi(M, t, v, i), \phi(M, t, v, i-1)$. Since the sequence

$$
\operatorname{Tor}_{i}^{R}\left(N^{\prime}, M_{-v^{\prime}}\right) \rightarrow \operatorname{Tor}_{i}^{R}\left(N, M_{-v^{\prime}}\right) \rightarrow \operatorname{Tor}_{i}^{R}\left(N^{\prime \prime}, M_{-v^{\prime}}\right) \rightarrow \operatorname{Tor}_{i-1}^{R}\left(N^{\prime}, M_{-v^{\prime}}\right)
$$

is exact, there is a set $S \subset \operatorname{Tor}_{i}^{R}\left(N, M_{-v^{\prime}}\right)$ that surjects onto

$$
\operatorname{ker}\left(\operatorname{Im}\left(\operatorname{Tor}_{i}^{R}\left(N^{\prime \prime}, M_{-v}\right)\right) \rightarrow \operatorname{Im}\left(\operatorname{Tor}_{i-1}^{R}\left(N^{\prime}, M_{-v}\right)\right)\right. \text {. }
$$

Let $I \subset \operatorname{Tor}_{i}^{R}\left(N, M_{-v^{\prime}}\right)$ be the image of $\operatorname{Tor}_{i}^{R}\left(N^{\prime}, M_{-v^{\prime}}\right) \rightarrow \operatorname{Tor}_{i}^{R}\left(N, M_{-v^{\prime}}\right)$. Since the sequence

$$
\operatorname{Tor}_{i}^{R}\left(N^{\prime}, M\right) \rightarrow \operatorname{Tor}_{i}^{R}(N, M) \rightarrow \operatorname{Tor}_{i}^{R}\left(N^{\prime \prime}, M\right) \rightarrow \operatorname{Tor}_{i-1}^{R}\left(N^{\prime}, M\right)
$$

is exact, the images of $I$ and $S$ in $\operatorname{Tor}_{i}^{R}(N, M)$ generate $\operatorname{Tor}_{i}^{R}(N, M)$.

8.5. Lemma. $\operatorname{Tor}_{i}^{R}\left(N, M^{*}\right)=\operatorname{Ext}_{R}^{i}(N, M)^{*}$, where $M$ and $N$ are finitely generated $R$-modules, and -* is the Matlis dual of -.

Proof. Let $L_{\bullet}$ be a free resolution of $N$, where each $L_{i}$ is finite. Tensoring $L_{\bullet}$ with $M^{*}$ produces the same complex as homing $L_{\bullet}$ into $M$, and then homing into $E$; hence the isomorphism.

8.6. Proposition. Assume $R$ is a Cohen-Macaulay ring which is locally Gorenstein on its punctured spectrum and $M=E / E_{-s}$. Then there exists $t$ such that for all finitely generated Cohen-Macaulay $R$-modules $N$, the $R$-modules $\operatorname{Tor}_{i}^{R}(N, M)$ are annihilated by $m^{t}$ for all $i \neq \operatorname{codim} N$.

Proof. $M=\left(m^{s}\right)^{*}$, so by Lemma 8.5, $\operatorname{Tor}_{i}^{R}(N, M)=\operatorname{Ext}_{R}^{i}\left(N, m^{s}\right)^{*}$, so it is enough to prove that the corresponding $\operatorname{Ext}_{R}^{i}\left(N, m^{s}\right)$ are annihilated by $m^{t}$. Let $r_{1}, \ldots, r_{n} \in m$ be a system of parameters of $R$ and let $\omega$ be the canonical module of $R$. Since $m_{r_{j}}^{s}=\omega_{r_{j}}$, there is an exact sequence $0 \rightarrow m^{s} \rightarrow \omega \rightarrow C_{j} \rightarrow 0$, where $C_{j}$ is annihilated by a power of $r_{j}$; raising $r_{j}$ to a suitable power we may assume that $C_{j}$ is annihilated by $r_{j}$. Since for every finitely generated Cohen-Macaulay $N$ we have that $\operatorname{Ext}_{R}^{i}(N, \omega)=0$ for all $i \neq \operatorname{codim} N$, the long exact sequences corresponding to the above short exact sequences imply that $\operatorname{Ext}_{R}^{i}\left(N, m^{s}\right)$, for $i \neq \operatorname{codim} N$, are annihilated by $r_{j}$ for all $j$, hence by $\left(r_{1}, \ldots, r_{n}\right)$, and we are done, since $\left(r_{1}, \ldots, r_{n}\right)$ is an $m$-primary ideal.

8.7. Proposition. Assume $R$ is a Cohen-Macaulay ring which is locally Gorenstein on its punctured spectrum. Let $M=E / E_{-s}$. Then for each $i>0$, there is $v$ such that for all $e$, the natural map $\operatorname{Tor}_{i}^{R}\left(R^{(e)}, M_{-v}\right) \rightarrow \operatorname{Tor}_{i}^{R}\left(R^{(e)}, M\right)$ is surjective.

Proof. Let $r_{1}, \ldots, r_{n}$ be as in the proof of Proposition 8.6, let $N=N_{0}=R^{(e)}$ and let $N_{t}=N / N\left(r_{1}, \ldots, r_{t}\right)$. Then $N_{t}$ is a right Cohen-Macaulay $R$-module, and it is 
enough to prove that for each $i>t$ there is $v$, depending only on $i, t$ (but not on $e)$, such that the natural map $\operatorname{Tor}_{i}^{R}\left(N_{t}, M_{-v}\right) \rightarrow \operatorname{Tor}_{i}^{R}\left(N_{t}, M\right)$ is surjective.

We use descending induction on $t$. If $t=n$, we are done by Proposition 8.4. We are going to show that the same $v$ works for all $t$. If $t<n$, we have the short exact sequence $0 \rightarrow N_{t} \stackrel{\times r_{t+1}}{\longrightarrow} N_{t} \rightarrow N_{t+1} \rightarrow 0$. Tensoring it with $M$ and considering that, by Proposition 8.6, $r_{t+1}$ annihilates $\operatorname{Tor}_{i}^{R}\left(N_{t}, M\right)$ for $i>t$, we get a surjection $\operatorname{Tor}_{i+1}^{R}\left(N_{t+1}, M\right) \rightarrow \operatorname{Tor}_{i}^{R}\left(N_{t}, M\right)$. Thus the bottom map in the commutative diagram

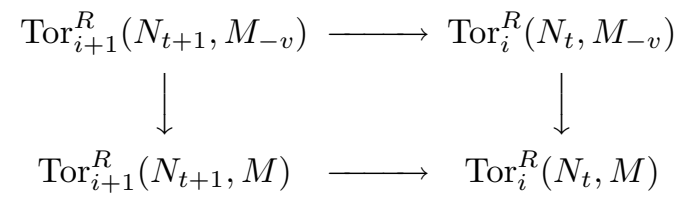

is surjective. The left hand vertical map is surjective by induction; hence the right hand vertical map is surjective as well.

8.8. Theorem. Assume $R$ is a Cohen-Macaulay ring which is locally Gorenstein on its punctured spectrum. Then $0_{E}^{*}=0_{E}^{* f g}$.

Proof. This follows from Lemma 8.3 and Proposition 8.7.

Let $T \subset R$ be a free $R^{p}$-module. This produces, for each $e$, a free $R^{p^{e}}$-submodule $T_{e} \subset R$ as follows. Let $T_{1}=T$. If $T_{1}, \ldots, T_{e-1}$ have been constructed, pick a free $R^{p^{e-1}}$-basis $\left\{t_{e, i}\right\}$ of $T_{e-1}$. Viewing $T_{1}$ as an $R^{p^{e+1}}$-submodule of $R^{p^{e}}$ (via the identification of $R^{p}$ with $R$ and $R^{p^{e+1}}$ with $R^{p}$ ), let $T_{1, e, i} \subset R^{p^{e-1}} t_{i}$ be the corresponding submodule of $R^{p^{e-1}} t_{e, i}$. We let $T_{e}$ be the union of $T_{1, e, i}$ over all $i$. It is unclear whether this construction depends on the choice of the $t_{i}$ 's.

Hence if $T \subset R^{(1)}$ is a free right $R$-submodule, then the above construction produces for each $e$ a free right $R$-submodule $T_{e} \subset R^{(e)}$.

8.9. Lemma. If $r \in R$ annihilates $R^{(1)} / T_{1}$, then $r^{2}$ annihilates $R^{(e)} / T_{e}$ for all e.

Proof. Viewing the right $R$-module $R^{(e)}$ as the $R^{p^{e}}$-module $R$, all we need to show is that $r^{2 p^{e}}$ annihilates $R / T_{e}$. We have the inclusions $R \supset T_{1} \supset T_{2} \supset \cdots \supset T_{e}$. Since $r$ annihilates $R / T_{1}$, we conclude that $r^{p^{j}}$ annihilates $R^{p^{j-1}} t_{j, i} / T_{1, j, i}$ for all $j, i$. Hence it annihilates $T_{j-1} / T_{j}$. Hence $r^{1+p^{2}+\cdots+p^{e}}=r^{\left(p^{e+1}-1\right) /(p-1)}$ annihilates $R / T_{e}$. But $\left(p^{e+1}-1\right) /(p-1)<2 p^{e}$, so $r^{2 p^{e}}$ annihilates $R / T_{e}$.

8.10. Proposition. Assume $R$ is an isolated singularity. Then there exists $t$ such that for all $e, i>0$ and all $R$-modules $M$, the right $R$-modules $\operatorname{Tor}_{i}^{R}\left(R^{(e)}, M\right)$ are annihilated by $m^{t}$.

Proof. Let $x_{1}, \ldots, x_{s}$ generate $m$. Since $R_{x_{i}}^{(1)}$, for every $i$, is a free right $R_{x_{i}}$-module, there exists a free right $R$-submodule $T_{1}$ of $R^{(1)}$ such that $R^{(1)} / T_{1}$ is annihilated by a power of $x_{i}$. Let $y_{i}$ be the square of that power of $x_{i}$. Then $y_{i}$ annihilates all $R^{(e)} / T_{e}$. The short exact sequence $0 \rightarrow T_{e} \rightarrow R^{(e)} \rightarrow R^{(e)} / T_{e} \rightarrow 0$ upon tensoring with $M$ produces a long exact sequence, which (considering that $\operatorname{Tor}_{i}^{R}\left(T_{e}, M\right)=0$ for $i>0$ ) shows that $\operatorname{Tor}_{i}^{R}\left(R^{(e)}, M\right)$, for all $i>0$, is a submodule of $\operatorname{Tor}_{i}^{R}\left(R^{(e)} / T_{e}, M\right)$. Since $R^{(e)} / T_{e}$ is annihilated by $y_{i}$, so is $\operatorname{Tor}_{i}^{R}\left(R^{(e)} / T_{e}, M\right)$, and hence so is $\operatorname{Tor}_{i}^{R}\left(R^{(e)}, M\right)$. Hence $\operatorname{Tor}_{i}^{R}\left(R^{(e)}, M\right)$ are annihilated by the ideal $\left(y_{1}, \ldots, y_{s}\right)$. But this ideal is $m$ primary, hence contains $m^{t}$ for some $t$. 
8.11. Proposition. Let $R$ be an isolated singularity. Let $M$ be an Artinian $R$ module. Then for each $i>0$ there is $v$ such that for all $e$, the natural map $\operatorname{Tor}_{i}^{R}\left(R^{(e)}, M_{-v}\right) \rightarrow \operatorname{Tor}_{i}^{R}\left(R^{(e)}, M\right)$ is surjective.

Proof. Let $I_{j} \subset R$ be the annihilator of $H_{m}^{j}(R)$. Since $R$ is Cohen-Macaulay on the punctured spectrum, it follows from local duality that $I_{j}$ is $m$-primary for $j<\operatorname{dim} R$. Hence $I=\bigcap_{j=0}^{\operatorname{dim} R-1} I_{j}$ is $m$-primary as well, and it annihilates all $H_{m}^{j}(R)$. Then $I$ annihilates $H_{m}^{j}\left(R^{(e)}\right)$ for every $e$, and every $j<\operatorname{dim} R$. So it is enough to prove the following statement:

Let $N$ be any finitely generated $R$-module satisfying the following three conditions:

(a) $N$ is locally free on the punctured spectrum of $R$.

(b) $I$ annihilates $H_{m}^{j}(N)$ for every $j<\operatorname{dim} R$.

(c) $\operatorname{Tor}_{i}^{R}\left(N, N^{\prime}\right)$, for all $R$-modules $N^{\prime}$ and all $i>0$, are annihilated by $m^{t}$.

Then for each $i$ there is $v$, depending only on $i, t$ and $I$ (but not on $N$ ), such that the natural map $\operatorname{Tor}_{i}^{R}\left(N, M_{-v}\right) \rightarrow \operatorname{Tor}_{i}^{R}(N, M)$ is surjective.

Let $r_{1}, \ldots, r_{d} \in R$ be a system of parameters, where $d=\operatorname{dim} R$. Let $N_{0}, \ldots, N_{d}$ be given inductively by $N_{0}=N$ and $N_{j+1}=N_{j} /\left(r_{j+1} N_{j}+\Gamma_{m}\left(N_{j}\right)\right)$ for $j \geq 0$.

It follows by induction on $j$ that $N_{j}$ is annihilated by the ideal $\left(r_{1}, \ldots, r_{j}\right)$. It follows from (a) that on the punctured spectrum $N_{j}$ is Cohen-Macaulay and $r_{1}, \ldots, r_{d}$ is a regular sequence, so the submodule of $N_{j}$ annihilated by $r_{j+1}$ is contained in $\Gamma_{m}\left(N_{j}\right)$, i.e. $r_{j+1}$ is a regular element on $N_{j} / \Gamma_{m}\left(N_{j}\right)$. The long exact local cohomology sequence associated with the short exact sequence

$$
0 \rightarrow \Gamma_{m}\left(N_{j}\right) \rightarrow N_{j} \rightarrow N_{j} / \Gamma_{m}\left(N_{j}\right) \rightarrow 0
$$

shows that $H_{m}^{s}\left(N_{j}\right)$, for all $s>0$, is isomorphic to $H_{m}^{s}\left(N_{j} / \Gamma_{m}\left(N_{j}\right)\right)$. This and the long exact local cohomology sequence associated with the short exact sequence

$$
0 \rightarrow N_{j} / \Gamma_{m}\left(N_{j}\right) \stackrel{r_{j+1}}{\longrightarrow} N_{j} / \Gamma_{m}\left(N_{j}\right) \rightarrow N_{j+1} \rightarrow 0
$$

imply that there exist exact sequences

$$
\begin{gathered}
0 \rightarrow H_{m}^{0}\left(N_{j+1}\right) \rightarrow H_{m}^{1}\left(N_{j}\right), \\
H_{m}^{s}\left(N_{j}\right) \rightarrow H_{m}^{s}\left(N_{j+1}\right) \rightarrow H_{m}^{s+1}\left(N_{j}\right) \text { for } s>0 .
\end{gathered}
$$

Hence $H_{m}^{s}\left(N_{j}\right)$ is annihilated by $I^{2^{j}}$, for this is true by assumption if $j=0$, and if $j>0$, then, by induction on $j, H_{m}^{s}\left(N_{j-1}\right)$ and $H_{m}^{s+1}\left(N_{j-1}\right)$ are annihilated by $I^{2^{j-1}}$, so by $\left.{ }^{* * *}\right), I^{2^{j-1}+2^{j-1}}=I^{2^{j}}$ annihilates $H_{m}^{s}\left(N_{j}\right)$.

We claim that, for all $N^{\prime}$, and all $i>j$,

(i) $\operatorname{Tor}_{i}^{R}\left(N_{j}, N^{\prime}\right)$ is annihilated by $I^{2^{j}(j+1)}$, and

(ii) $\operatorname{Tor}_{i}^{R}\left(N_{j} / \Gamma_{m}\left(N_{j}\right), N^{\prime}\right)$ is annihilated by $I^{2^{j}(j+2)}$.

Indeed, (i) holds for $j=0$ by assumption. If (i) holds for some $j$, then $\left(^{*}\right)$ produces exact sequences $\operatorname{Tor}_{i}^{R}\left(N_{j}, N^{\prime}\right) \rightarrow \operatorname{Tor}_{i}^{R}\left(N_{j} / \Gamma_{m}\left(N_{j}\right), N^{\prime}\right) \rightarrow \operatorname{Tor}_{i-1}^{R}\left(\Gamma_{m}\left(N_{j}\right), N^{\prime}\right)$ for all $i$. Since $I^{2^{j}}$ annihilates $\Gamma_{m}\left(N_{j}\right)$, it annihilates $\operatorname{Tor}_{i-1}^{R}\left(\Gamma_{m}\left(N_{j}\right), N^{\prime}\right)$. Hence $I^{2^{j}(j+2)}$, for $i>j$, annihilates $\operatorname{Tor}_{i}^{R}\left(N_{j} / \Gamma_{m}\left(N_{j}\right), N^{\prime}\right)$. Thus if (i) holds for some $j$, then (ii) also holds for the same $j$.

By $(* *), \operatorname{Tor}_{i+1}^{R}\left(N_{j} / \Gamma_{m}\left(N_{j}\right), N^{\prime}\right) \rightarrow \operatorname{Tor}_{i+1}^{R}\left(N_{j+1}, N^{\prime}\right) \rightarrow \operatorname{Tor}_{i}^{R}\left(N_{j} / \Gamma_{m}\left(N_{j}\right), N^{\prime}\right)$ is exact, which implies that if (ii) holds for $j$, then (i) holds for $j+1$. This completes the proof of the claim. 
By taking all $r_{1}, \ldots, r_{d}$ from the ideal $I^{2^{d}(d+1)}$ we can ensure that $r_{j+1}$ annihilates $\operatorname{Tor}_{i}^{R}\left(N_{j} / \Gamma_{m}\left(N_{j}\right), N^{\prime}\right)$.

We claim that for each $i>j$ there are $v$ and $v^{\prime}>v$, depending only on $i, j, t$ and $r_{1}, \ldots, r_{d}$ (but not on $N$ ), such that the natural map $\operatorname{Tor}_{i}^{R}\left(N_{j}, M_{-v^{\prime}}\right) \rightarrow$ $\operatorname{Tor}_{i}^{R}\left(N_{j}, M\right)$ induces an isomorphism between the images of $\operatorname{Tor}_{i}^{R}\left(N_{j}, M_{-v}\right)$ in both modules. Indeed, if $j=d$, then $N_{d}=\Gamma_{m}\left(N_{d}\right)$ is annihilated by $I^{2^{d}}$, so we are done by Proposition 8.4. If $j<d$, then $r_{j+1}$ annihilates $\operatorname{Tor}_{i}^{R}\left(N_{j} / \Gamma_{m}\left(N_{j}\right), N^{\prime}\right)$ for all $i>j$ and all $N^{\prime}$, so the long exact sequences associated with $(* *)$ produce the following commutative diagrams for all $i>j$ and $v^{\prime}>v$ (where $\bar{N}_{j}=N_{j} / \Gamma_{m}\left(N_{j}\right)$ and $\mathrm{Im}$ means the image in the corresponding $\operatorname{Tor}_{*}^{R}\left(-, M_{-v^{\prime}}\right)$ :

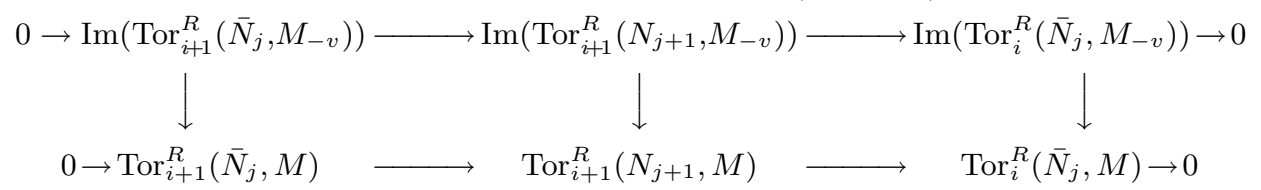

The bottom row is exact, and the top row is exact in the left and right terms. By descending induction on $j$, there are $v$ and $v^{\prime}>v$ such that the middle vertical map is bijective; hence the vertical map on the right is surjective. Increasing $v$ and $v^{\prime}$, if necessary, we can assume that they work for $i+1$ as well, i.e. the vertical map on the left is surjective as well. Since the middle vertical map is bijective, both vertical maps on the left and on the right are bijective as well.

(*) implies that for all $v^{\prime \prime} \geq v^{\prime}$ there exists the following commutative diagram, where $\operatorname{Im}$ means the image in the corresponding $\operatorname{Tor}_{*}^{R}\left(-, M_{-v^{\prime \prime}}\right)$ :

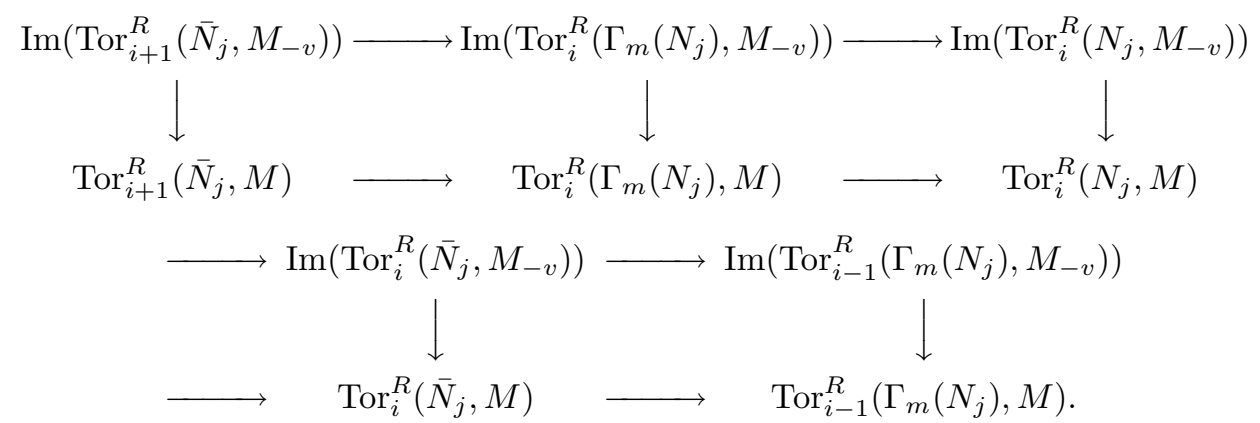

It follows from above remarks and Proposition 8.4 that there exists $v^{\prime}$ such that for all $v^{\prime \prime} \geq v^{\prime}$, the two vertical maps on the left and the two on the right are bijective. This plus the exactness of the bottom row immediately implies that the top row is exact in the second term from the left. Since the sequence $\operatorname{Tor}_{i}^{R}\left(N_{j}, M_{-v^{\prime}}\right) \rightarrow$ $\operatorname{Tor}_{i}^{R}\left(\bar{N}_{j}, M_{-v^{\prime}}\right) \rightarrow \operatorname{Tor}_{i-1}^{R}\left(\Gamma_{m}\left(N_{j}\right), M_{-v^{\prime}}\right)$ is exact, there is $S \subset \operatorname{Tor}_{i}^{R}\left(N_{j}, M_{-v^{\prime}}\right)$ that surjects onto $\operatorname{ker}\left(\operatorname{Im}\left(\operatorname{Tor}_{i}^{R}\left(\bar{N}_{j}, M_{-v}\right)\right) \rightarrow \operatorname{Im}\left(\operatorname{Tor}_{i-1}^{R}\left(\Gamma_{m}\left(N_{j}\right), M_{-v}\right)\right)\right.$ ), where Im means the image in $\operatorname{Tor}_{*}^{R}\left(-, M_{-v^{\prime}}\right)$. Replacing $v$ by $v^{\prime}$ and picking an appropriate new $v^{\prime}$, we see that the image of $S$ in the middle term of the top row surjects onto the kernel of the top horizontal right hand map, i.e. the top row is exact in the second term from the right. Since the sequence $\operatorname{Tor}_{i}^{R}\left(\Gamma_{m}\left(N_{j}\right), M_{-v^{\prime}}\right) \rightarrow$ $\operatorname{Tor}_{i}^{R}\left(N_{j}, M_{-v^{\prime}}\right) \rightarrow \operatorname{Tor}_{i}^{R}\left(\bar{N}_{j}, M_{-v^{\prime}}\right)$ is exact, there is $S \subset \operatorname{Tor}_{i}^{R}\left(\Gamma_{m}\left(N_{j}\right), M_{-v^{\prime}}\right)$ that surjects onto $\operatorname{ker}\left(\operatorname{Im}\left(\operatorname{Tor}_{i}^{R}\left(N_{j}, M_{-v}\right)\right) \rightarrow \operatorname{Im}\left(\operatorname{Tor}_{i}^{R}\left(\bar{N}_{j}, M_{-v}\right)\right)\right)$, where Im means the image in $\operatorname{Tor}_{i}^{R}\left(-, M_{-v^{\prime}}\right)$. Replacing $v$ by $v^{\prime}$ and picking an appropriate new $v^{\prime}$, we see that the image of $S$ in the second term from the left of the top row surjects onto the kernel of the second horizontal map from the right in the top row. Hence 
the top row is exact and by the 5-lemma the middle vertical map is bijective. All the choices of $v$ and $v^{\prime}$ we have made in this argument depend only on $i, j, t$ and $r_{1}, \ldots, r_{d}$, but not on $N$. This completes the proof of the claim.

The proposition follows from the $j=0$ case of the claim.

8.12. Theorem. If $R$ is an isolated singularity, then $0_{M}^{*}=0^{* f g}$ for every Artinian $R$-module $M$.

Proof. This follows from Proposition 8.11 and Lemma 8.3.

\section{REFERENCES}

[AM] Aberbach, I., and MacCrimmon, B., Some results on test ideals, Proc. Edinburgh Math. Soc. (2) 42 (1999), 541-549. CMP 2000:04

[A1] Aoyama, Y., On the depth and the projective dimension of the canonical module, Japan. J. Math.(N.S.) 6 (1980), 61-66. MR 82h:13007

[A2] Aoyama, Y., Some basic results on canonical modules, J. Math. Kyoto Univ. 23 (1983), 85-94. MR 84i:13015

[C] Vassilev, J., Test ideals in quotients of F-finite regular local rings, Trans. Amer. Math. Soc. 350 (1998), 4041-4051. MR 98m:13009

[G] Glassbrenner, D., Strong F-regularity in images of regular rings, Proceedings of AMS 124 (1996), 345-353. MR 96d:13004

[Ha] Hartshorne, R., Algebraic Geometry, Springer-Verlag, New York, 1993. MR 57:3116 (1st ed.)

[HH1] Hochster, M. and Huneke, C., Tight closure, invariant theory, and the Briancon-Skoda theorem, JAMS 3 (1990), 31-116. MR 91g:13010

[HH2] Hochster, M. and Huneke, C., Tight closure and strong F-regularity, Mémoires de la Société Mathématique de France 38 (1989), 119-133. MR 91i:13025]

[HH3] Hochster, M. and Huneke, C., F-regularity, test elements and smooth base change, Trans. Amer. Math. Soc. 346 (1994), 1-62. MR 95d:13007

[HH4] Hochster, M. and Huneke, C., Applications of the Existence of Big Cohen-Macaulay Algebras, Advances in Math 113 (1995), 45-117. MR 96d:13014

[HH5] Hochster, M. and Huneke, C., Indecomposable canonical modules and connectedness, in Commutative Algebra: Syzygies, Multiplicities, and Birational Algebra, eds. Heinzer, Huneke, Sally, Contemporary Mathematics 159 (1994), 197-208. MR 95e:13014

[Ku] Kunz, E., On Noetherian rings of characteristic p, Amer. J. Math. 97 (1975), 791-813. MR 55:5612

[L] Lyubeznik, G., F-modules: Applications to local cohomology and D-modules in characteristic $p>0$, J. f. d. reine u. angew. Math. 491 (1997), 65-130. MR 99c:13005

[LS] Lyubeznik, G. and Smith, K., Strong and weak F-regularity are equivalent for graded rings, American Journal of Mathematics 121 (1999), 1279-1290. CMP 2000:05

[Mac] MacCrimmon, B., Strong F-regularity and boundedness questions in tight closure, University of Michigan, thesis (1996).

[Mats] Matsumura, H., Commutative Ring Theory, Cambridge University Press, Cambridge, 1989. MR 90i:13001

[Na] Nagata, M., Local Rings, Interscience Publishers (a division of John Wiley and Sons), New York, 1962. MR 27:5790

[P1] Popescu, D., General Néron desingularization, Nagoya Math. J. 100 (1985), 97-126. MR 87f:13019

[P2] Popescu, D., General Néron desingularization and approxiation, Nagoya Math. J. 104 (1986), 85-115. MR 88a:14007

[R] Radu, N., Une classe d'anneaux noetheriens, Rev. Roumaine Math. Pures Appl. 37 (1992), 79-82. MR 93g:13014

[S1] Smith, Karen E., Tight closure of parameter ideals and F-rationality, University of Michigan, thesis (1993).

[S2] Smith, Karen E., Tight closure of parameter ideals, Invent. Math. 115 (1994), 41-60. MR 94k:13006 
[S3] Smith, Karen E., Test ideals in local rings, Trans. Amer. Math. Soc. 347 (9) (1995), 3453-3472. MR 96c:13008

[S4] Smith, Karen E., F-rational rings have rational singularities, Amer. Jour. Math. 119 (1) (1997), 159-180. MR 97k:13004

[Sw] Swan, R., Néron-Popescu desingularization, Algebra and Geometry (Taipei, 1995), Lect. Algebra Geom., vol. 2, International Press, Cambridge, MA, 135-192. MR 2000h:13006

[W] Williams, L., Uniform stability of kernels of Koszul cohomology indexed by the Frobenius endomorphism, Jour. of Alg. 172 (1995), 721-743. MR 96f:13003

Department of Mathematics, University of Minnesota, Minneapolis, Minnesota 55455

E-mail address: gennady@math.umn.edu

Department of Mathematics, University of Michigan, Ann Arbor, Michigan 48109

E-mail address: kesmith@math.lsa.umich.edu 